\title{
A comprehensive approach for the appraisal of the barrier effect of roads on pedestrians
}

\author{
Paulo Anciaes \\ Peter Jones \\ University College London, United Kingdom
}

Published in Transportation Research Part A: Policy and Practice 134, 227-250

\begin{abstract}
Roads can become physical and/or psychological barriers to the movement of pedestrians, an impact known as the "barrier effect" or "community severance". This paper proposes a new approach for measuring and valuing the barrier effect of different types of roads and for integrating the values into the appraisal of transport projects. This approach was developed based on the results of a survey of residents in areas around busy roads in two English cities. A series of stated preference exercises elicited preferences regarding crossing roads with specified design and traffic characteristics in locations with or without designated crossing facilities and making trade-offs with walking time and benefits or costs. The exercises were customised to represent different trip purposes (work, shopping, or leisure). Results were scaled with those obtained from a revealed preference exercise among some of the same participants, who indicated on a map their usual walking routes to locations that required them to cross the road. The results of the models of the participants' choices were then used to develop an index of the size of the barrier effect caused by the different characteristics of roads (number of lanes, presence/width of central reservation (median strip), traffic density, and traffic speed) and pedestrian crossing facilities (type, waiting time, and walking time to access them). The index was also related with the estimated willingness to pay to reduce the barrier effect for existing trips, and with the number of new walking trips that could be generated with that reduction.
\end{abstract}

\section{Introduction}

\subsection{State of the art}

Road infrastructure and motorised road traffic often become physical and psychological barriers to the movement of pedestrians, a phenomenon known in the literature as the "barrier 
effect" of roads (Lerväg 1984; Soguel 1995; Russell and Hine 1996; Héran 2011; Anciaes et al. 2016a, 2016b; Van Eldijk 2018). In some cases, this happens because of the presence of physical structures (such as walls or guard railings) preventing pedestrians from crossing to the other side. In other cases, crossing is difficult because of the width of the road or the number or speed of vehicles. Pedestrian crossing facilities often aggravate, rather than mitigate, the problem, due to their poor design and maintenance or the effort and inconvenience of using them (Rankavat and Tiwari, 2016; Räsänen et al. 2007; Tao et al. 2010). Figure 1 shows examples of barriers caused by roads and traffic (left side) and crossing facilities (right side).

\section{Figure 1: Examples of the barrier effect of roads on pedestrians}
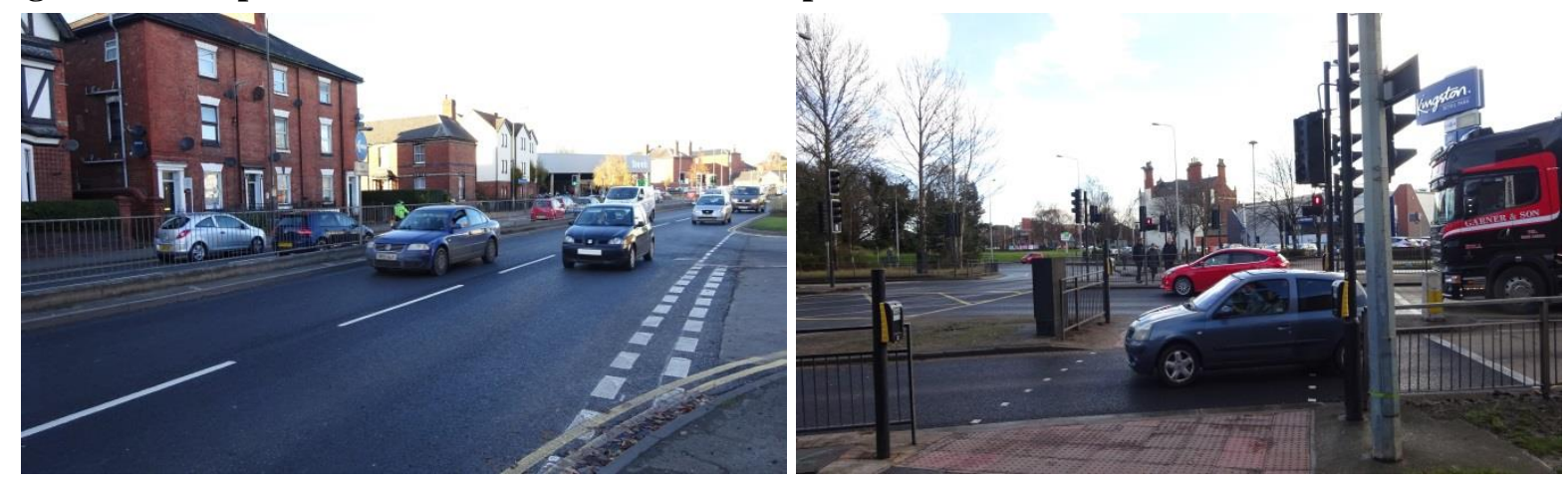

Left: Hereford, UK; Right: Hull, UK.

The barrier effect of roads may lead to a chain of negative direct and indirect impacts on individuals and communities (Anciaes et al. 2016a). At a first level, the barriers cause detours, delays to walking trips and increase the risk of vehicle-pedestrian collisions (Hine 1996, Jacobsen et al. 2009, Granié et al. 2014) and the unpleasantness of crossing the road due to the exposure to traffic noise and air pollution and feelings of intimidation (James et al. 2005, Villaveces et al. 2012). These effects then contribute to the separation of communities from other communities and potential trip destinations (Handy 2003; James et al. 2005; Héran 2011; Anciaes et al. 2016a, 2016b; Mindell et al. 2017) and possibly to wider negative impacts on levels of physical activity, health, wellbeing, and social cohesion (Mindell and Karlsen 2012; Foley et al. 2017; Mindell et al. 2017; Nimegeer et al. 2018; Anciaes et al. 2019). In particular, there is consistent evidence that roads with high traffic volumes and/or speeds reduce levels of social interaction (Appleyard and Lintell 1972; Bosselmann et al. 1999; Sauter and Huettenmoser 2008).

The barrier effect is often described in the literature using the concept of "community severance". A review of 60 definitions of "barrier effect" and "community severance" (Anciaes 2015) found that the two terms are often used interchangeably, although studies 
looking at "community severance" tend to put more emphasis on the wider impacts on local communities. In the current paper we focus on the narrower concept of "barrier effect" of roads on the movement of pedestrians and not on its potential wider impacts.

There are few methods to measure and value the barrier effect of roads and the benefits of policy interventions to reduce that effect (Anciaes et al. 2016b). In most countries, the barrier effect is either not mentioned in guidance documents for transport appraisal or described in broad terms, without suggesting practical methods to quantitatively assess the effect (Anciaes et al. 2016b). Guidance documents in a few countries suggest using simple methods such as qualitative scales (UK DfT 2017, Ch.5) or indicators based on the extent of pedestrian detour (Ecoplan 2010, Ch.6U141) or pedestrian delay (BMVI 2016; MIT 2008; ATAP 2018). In the latter case, the effect can be monetised by multiplying the delay by the unit value of time for walking trips. However, these methods are based on expert assessments and not on information about the preferences of pedestrians. In addition, they do not isolate the cost of the barrier effect caused by different characteristics of the roads, traffic, and crossing facilities, and do not value the impacts on suppression of walking trips.

Over the years, researchers have proposed several methods to measure the barrier effect of roads, but have focused on partial aspects of the problem and left unanswered questions regarding how to use the outputs of those methods in project appraisal. Proposals include objective measures of road design and traffic regulations (Baltes and Chu 2002), accessibility indicators (Van Eldijk 2018), catchment areas for walking trips (Héran 2011), pedestrian crossing behaviour (Russell and Hine 1996), perceptions of safety (Tate 1995), and the size of one's personal neighbourhood (Lassière 1976). A few studies commissioned by transport authorities have proposed fairly complex approaches (Clark et al. 1991; Tate 1997; Read and Cramphorn 2001), but there is little evidence of these approaches ever being used in practice.

Recently, researchers have started to estimate the monetary value of reducing the barrier effect based on people's perceived disutility regarding different aspects of the road. In a previous paper (Anciaes et al. 2018), we developed a stated preference study to estimate the unit value of marginal improvements in road design and traffic conditions for pedestrians crossing the road in locations with no crossing facilities, and explored issues such as nontrading behaviour, reasons for choices, and sensitivity of values to demographic characteristics. However, the analysis was based on a simple single stated preference exercise that captured only a part of the barrier effect of roads, as it did not consider the presence and 
type of pedestrian crossing facilities, differences by trip purpose, or the effect on the number of walking trips across the road.

Similar gaps can be identified in other efforts to estimate the value of the barrier effect with stated preference techniques. For example, Soguel (1995) and Grisolía et al. (2015) estimated willingness to pay for road tunnels that completely remove the barrier effect, but did not consider less radical solutions to mitigate, rather than remove, the barrier effect by changing the characteristics of roads, traffic, or crossing facilities. Garrod et al. (2002) valued improvements to mitigate the barrier effect but framed the trade-off scenarios as a mix of causes (e.g. traffic speed) and first-level effects (e.g. time to cross the road and traffic noise), which fitted well with the objective of the study (valuing traffic calming policies) but it is difficult to translate into a framework for the appraisal of the barrier effect.

A parallel strand of the literature has used revealed preference methods to link some aspects of the barrier effect of roads with outcomes of local property markets. A few studies have estimated statistical associations between property prices and single characteristics of roads and/or traffic, usually traffic volumes (Kawamura and Mahajan 2005; Li and Saphores 2012; Allen et al. 2015). However, those characteristics may be capitalized by markets due to their effect on other problems, such as noise and air pollution, and not due to their effect on the movement of pedestrians. On the other hand, studies focusing on issues specifically affecting pedestrians, such as those relating property prices with traffic calming measures, capture market capitalization that is the result of the preferences of both local pedestrians and car users. This may explain why these studies have failed to find a positive willingness to pay for those measures (see for example Bretherton et al. 2000 and Graham and Jones 2019).

Notwithstanding the shortcomings of the specific methods detailed above, the use of stated preference and revealed preference methods in isolation is also limited by the nature of those methods. Stated preference surveys capture choices made in hypothetical settings and so they are useful for the assessment of planned improvements to reduce the barrier effect. However, the use of hypothetical scenarios limits the validity of the results, as it fails to consider people's behaviour in the real world. In contrast, revealed preference methods have the advantage of being based on actual behaviour (or the outcomes of that behaviour). However, by relying on specific case studies, these methods do not provide the necessary variation to allow predictions of the impact of possible policy interventions on different aspects of the problem. The valuation of the barrier effect of roads therefore requires the integration of 
stated preference and revealed preference methods, combining the advantages of the two methods.

As evident in this review, previous efforts to value the barrier effect of roads have captured only partial aspects of the problem, not taking into account that the barrier effect is caused by the interplay of three main aspects (road infrastructure, motorised traffic, and crossing facilities) and have consequences not only on the utility of existing walking trips but also on the number of suppressed trips. In addition, there are methodological issues derived from using stated preference and revealed preference methods in isolation and in a simplified way, not considering the range of different contexts that influence how barriers affect individuals.

\subsection{Contribution and objectives}

The main contribution of this paper is to address the gaps identified above by developing a comprehensive approach to measure and value the barrier effect of roads and of interventions to reduce that effect. We propose an index of the barrier effect caused by the different characteristics of roads (number of lanes, presence/width of central reservation (median strip), traffic density, and traffic speed), and pedestrian crossing facilities (type, waiting time, and walking time to access them). The index is then related to monetary values and with the number of suppressed trips across the road, i.e. the number of new trips that would be made if the barrier effect was reduced.

The second contribution of the paper is methodological, by using revealed preference to ensure that the stated preference results are consistent with real-world walking behaviour. We also investigate how the barrier effect is influenced by the context, by customizing the stated preference exercises to represent different trip purposes (work, shopping, or leisure), times of day (daytime and night-time) and trade-off situations (some involving benefits and other costs).

The paper proceeds as follows. The next section gives an overview of the survey questionnaire and describes the case study areas and survey methods. Sections 3 and 4 present the design of the stated and revealed preference exercises and the specification and results of the models of participants' choices. Section 5 describes the approach to apply the results in the appraisal of road projects. Section 6 concludes the paper.

\section{Survey questionnaire, case studies, and sample composition}

The research uses data captured with a newly developed survey questionnaire, described in detail in the sections that follow. The main part of this questionnaire comprised a series of 
three stated preference exercises to elicit preferences regarding crossing roads with specified design and traffic characteristics in locations with or without designated crossing facilities, based on trade-offs with walking time and personal benefits or costs. This was followed by a revealed preference exercise where participants indicated on a map their usual walking route for trips across the road for different purposes. Information about the demographic characteristics of participants was also collected.

The questionnaire was applied in the areas surrounding major roads in two English cities: the A49 road in Hereford and the A63/Ferensway roads in (Kingston upon) Hull. Hereford is a mid-sized city (population=60,415) in the West Midlands, close to the Welsh border. Hull is a larger port city (population=284,321) in the Northeast. The case study roads were chosen, among other possible roads in other English cities, because they are part of the Highways England Strategic Network (and thus play a major role at the national level), but cross through the central parts of the two cities. In Hereford, the A49 separates residential areas from the main shopping and services areas. In Hull, Ferensway separates residential areas from the main shopping and services areas and the A63 separates both from the waterfront leisure areas.

The case study roads have 2 or 3 lanes for motorised traffic in each direction (depending on the section), and high traffic volumes and speeds. The A49 in Hereford has a 30mph speed limit and traffic volumes varying from around 17,500 to 45,000 vehicles/day, depending on the section. The A63 in Hull has a 40mph speed limit and traffic volumes of around 50,000 vehicles/day. Ferensway has a 30mph limit and traffic volumes varying from around 16,500 to 21,000 vehicles/day. Crossing the road is not possible in some sections due to the presence of guard railings (see an example in Figure 1, left). Only a few sections have a central reservation. Most of the existing pedestrian crossing facilities are multiple-step staggered signalised crossings (see an example in Figure 1, right), where waiting time is in some cases above 2 minutes, or simple "pedestrian refuges", consisting of coloured tactile pavements on both kerbs, and in some cases, also in the central reservation. In some sections, the only crossing facility is an underpass or footbridge. In the Hull case study, there are also plans for a new high-quality covered footbridge with an innovative design.

The samples were drawn from the areas within walking distance from the road (defined as 400m). The Hereford and Hull study areas had 6597 and 7781 adult residents, respectively, at the time of the most recent population census (2011). Sampling quotas were imposed to achieve a reasonable sex and age balance. Recruitment was done door-to-door. Only one adult 
participant was recruited from each household. Participants were offered a $£ 10$ voucher as an incentive to participate. The interviews were conducted face-to-face during December 2017May 2018 in the participants' homes, using tablet computers.

The samples consisted of 305 participants in Hereford and 348 in Hull. Table 1 shows the main demographic characteristics of the two samples (columns 1 and 5), in comparison with the populations from which they were drawn (columns 3 and 6). Column 2 shows the characteristics of the subset of the Hereford sample that was used in the revealed preference analysis, as explained later in Section 4 of the paper. The samples are well aligned with the respective populations, with only a slight underrepresentation of individuals aged 16-34, and in Hull, also of men and individuals in 1-person households. The table also includes the characteristics of the whole population of Hereford and Hull (columns 4 and 7) and the urban population in England (column 8). The population in the Hull case study area has a higher proportion of no-car households and 1-person households and a slightly higher proportion of individuals with low qualifications than the whole Hull and England populations. 
Table 1: Sample characteristics in comparison with population of case studies, cities, and urban England

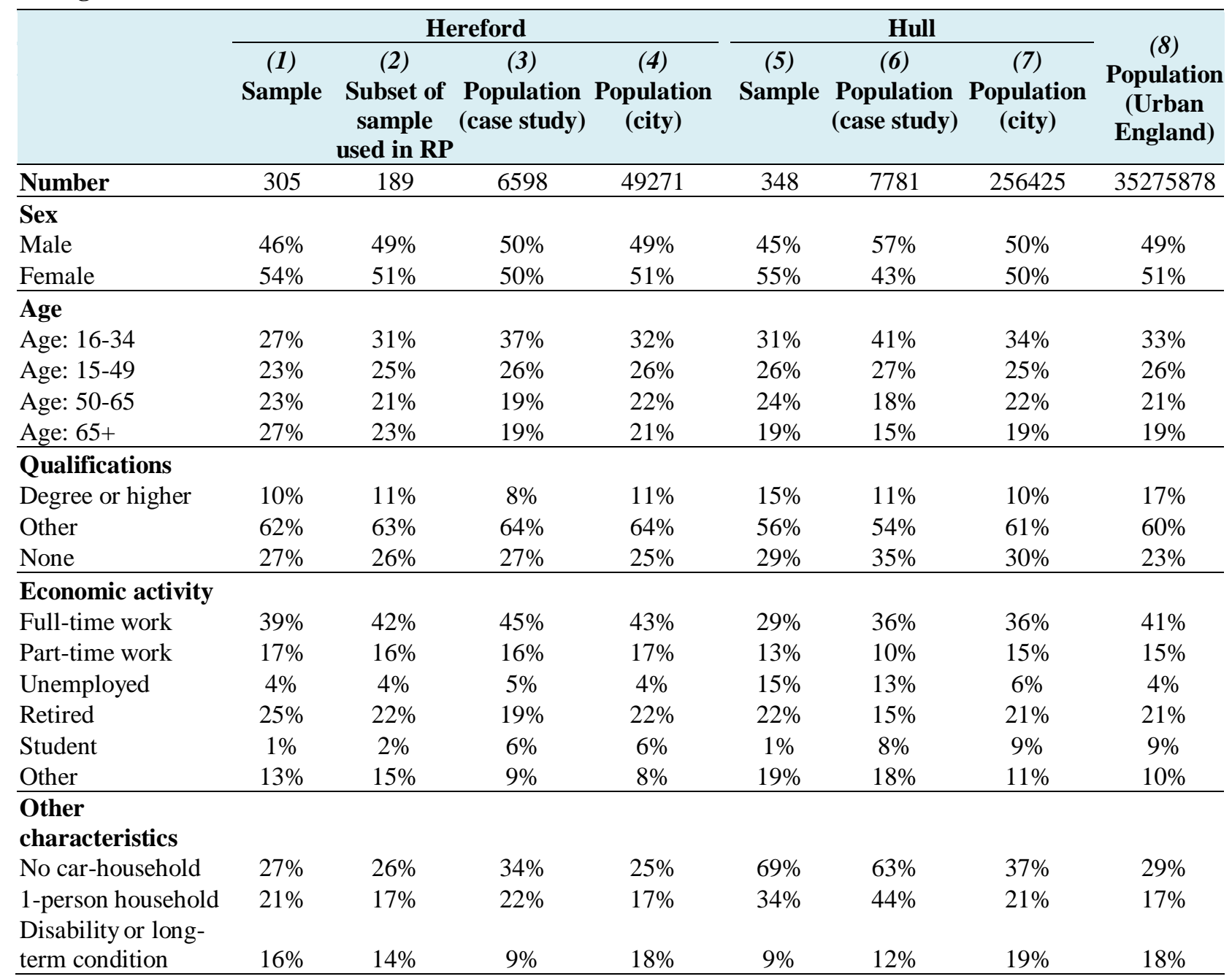

Note: Population includes only individuals aged 16 years or more. RP: revealed preference

\section{Stated preference exercises}

The stated preference component of the survey consisted of three exercises, synthesized in Figure 2 and further elaborated in the following subsections. The scenarios were defined in terms of trade-offs between walking time or monetary costs/benefits versus attributes defining the causes of the barrier effect (i.e. the characteristics of roads, traffic, and crossing facilities), rather than attributes defining its various levels of consequences described in the introduction of the paper (e.g. collision risk, unpleasantness of crossing the road, and wider impacts on local communities). 
Figure 2: Stated preference exercises: objective, number of participants, options, attributes, and levels

\begin{tabular}{|c|} 
SP1 \\
Informal crossing vs. walking time \\
500 participants \\
(97 work, 210 shopping, 193 leisure) \\
- Option A: Informal crossing \\
Number of lanes (1, 2, 3) \\
Central reservation (wide, narrow, no) \\
Traffic density (low, medium, high) \\
Traffic speed (10,20,30 or 20,30,40 mph) \\
- Option B: Walk to pedestrian platform \\
Additional walking time $(2,4, \ldots ., 20$ mins.) \\
- Option C: Do not make trip \\
\end{tabular}

\begin{tabular}{|c|}
\hline SP2 \\
Informal crossing vs. benefit/cost \\
533 participants \\
(102 work, 233 shopping, 198 leisure) \\
Version1 \\
- Option A: Informal crossing and benefit \\
Same attributes/levels as SP1 Option A \\
Benefit (f0.40, fO.80,... 4 ) \\
- Option B: Do not cross \\
Version2 \\
- Option A: Informal crossing \\
Same attributes/levels as SP1 Option A \\
- Option B: Do not cross and cost \\
Cost (f0.40, $£ 0.80, \ldots . .44)$
\end{tabular}

\begin{tabular}{|l|}
\hline \multicolumn{1}{|c|}{ SP3 } \\
Formal crossing vs. walking time \\
$\quad 653$ participants \\
(116 work, 289 shopping, 248 leisure) \\
- Options A and B: Formal crossing \\
Pedestrian refuge \\
Straight signalised \\
Staggered signalised \\
Footbridge \\
High-quality footbridge \\
Underpass \\
- Option C: Walk to pedestrian platform \\
Additional walking time $(2,4, \ldots . ., 20$ mins.) \\
- Option D: Do not make trip \\
\hline
\end{tabular}

The specific attributes and levels included in the exercises were chosen on the basis of the results of 4 focus group discussions and 7 in-depth interviews with individuals living near busy roads in England and representing different age and socio-economic groups. The set of attributes and levels was then refined with insights from various pilot studies and discussions in workshops with researchers and practitioners and in meetings with the national transport authority (Highways England) and the local authorities in the two case study areas. The attributes provides a good representation of the causes of the barrier effect (i.e. the design and traffic characteristics of roads and the type of crossing facilities). Traffic composition (e.g. proportion of large vehicles) was not included as an attribute as it was considered by participants in focus groups and interviews to be of less relevance than traffic density and speed.

The Ngene software was used to generate efficient designs, i.e. designs that minimize the standard errors of the model estimates. Priors were used from a pilot study in two areas near busy roads in London (Anciaes and Jones 2018). The design consisted of 12 blocks of 8 questions. However, as will be mentioned in Subsection 3.1., two extra questions were added to the SP1 exercise representing night-time scenarios.

To ensure that the attributes and levels of the stated preference exercises were well understood, before the exercises participants were asked a set of questions about the characteristics of the case study road and then shown an illustration (of the same type shown in the exercises) of the road as they had described it. The question about speeds included short videos of traffic, each one representing the same road and number of vehicles, but with vehicles moving at different speeds. Before the crossing facilities exercise (SP3), participants were also shown illustrations (of the same type shown in the exercises) and photos (taken in the case study road) of crossing facilities. Feedback questions after the exercises revealed that 
98\%-100\% of participants felt able to make comparisons between the options and found the scenarios described realistic.

\subsection{SP1: informal crossing vs. walking time}

\section{Design}

The first stated preference exercise estimated trade-offs between walking time and crossing a road in a location with no designated crossing facilities, and walking further to use some type of crossing facility. Three options were presented in each question (see example in Figure 3):

- Option A: Cross a road with specified design and traffic characteristics in a location with no designated crossing facilities

- Option B: Walk a specified number of extra minutes in order to cross in a pedestrian platform, i.e. a location where the road goes through a tunnel

- Option C: Do not make the trip

Figure 3: SP1 - example of question

Now please look at this screen. Looking at the road conditions on the left, which of the three options would you choose?

\begin{tabular}{|l|l|}
\hline Number of lanes in each direction & 2 \\
\hline Central reservation? & Not present \\
\hline Traffic density & Medium \\
\hline Traffic speed & $30 \mathrm{mph}$ \\
\hline
\end{tabular}

\begin{tabular}{|c|c|c|}
\hline Option A & Option B & Option C \\
\hline & Use covered over road & Don't make this trip \\
\hline (not at pedestrian crossing) & Adds 8 minutes to your journey & \\
\hline
\end{tabular}

Ooption A

Option B

Ooption C

The attributes of the problem were the additional walking time in Option B (ranging from 2 to 20 minutes, in increments of 2 minutes) and the characteristics of the road in Option A, i.e. the number of lanes for motorised traffic $(1,2$, or 3 in each direction), the existence/width of a central reservation (wide, narrow, or none), traffic density (low, medium, or high), and traffic speed $(10,20$, or $30 \mathrm{mph}$ for participants living near road sections where the speed limit is $30 \mathrm{mph}$; and 20,30 , or $40 \mathrm{mph}$ for those living in road sections where the speed limit is 40mph). A constraint was applied so that scenarios with high traffic density did not have the highest speed value, in order to account for road congestion. 
The SP1 exercise consisted of ten questions, each one presenting different attribute levels. The two last questions showed images with a darkened background, representing night-time scenarios.

The text introducing the exercise was customized so that participants responded in the context of a specific purpose for the walking trip. This purpose was assigned based on the answers to preliminary questions about the location of places associated with each trip purpose and the participants' usual travel behaviour. Participants who indicated in those questions that shops or supermarkets were within walking distance (either on their side or the other side of the road) and that they currently go there (by any means of transport) were identified as potentially making shopping trips. The same assessment was done for work trips (considering workplaces) and leisure trips (considering park/playing fields, community centres/leisure centres, pubs/restaurants/cafés, and houses of friends/neighbours/relatives). Participants were then randomly allocated to one of their potential trip purposes.

The exercise was designed to remove non-trading behaviour. This was done by including two preliminary choice questions, one showing the worst possible road in Option A and the minimum walking time in Option B, and another showing the best possible road in Option A and the maximum walking time in Option B. The $2 \%$ of participants who chose Option A in the first question and the $21 \%$ who did not choose Option $\mathrm{A}$ in the second question were identified as non-traders (i.e. those who would always choose to cross or always choose not to cross the road) and did not proceed to the exercise. Among the participants who completed the exercise, and across all questions, the frequencies of choices for Options A, B and C were $27 \%, 62 \%$ and $11 \%$ respectively.

\section{Model}

Choices were modelled using a mixed logit specification (McFadden and Train 2000). The utility $U_{i j q}$ for participant $i$ of option $j$ in question $q$ was assumed to depend on a vector of observed variables $\left(x_{i j q}\right)$, a vector $\beta_{i}$ of coefficients specific to each participant but constant across questions, and an error term $\varepsilon_{i j q}$, independent and identically distributed, following the Extreme Value Type 1 distribution. The coefficients $\beta_{i}$ vary in the population with density $\mathrm{g}\left(\beta_{i} \mid \theta\right)$, where $\theta$ represents the parameters of the distribution.

$$
U_{i j q}=\beta_{i} x_{i j q}+\varepsilon_{i j q}
$$

Participant $i$ chooses option $j$ in question $q$ if $U_{i j q}>U_{i k q}$, with $k$ denoting all other options. It can be shown that the probability of that choice, conditional on $\beta_{i}$ can be expressed in terms of a logistic function, as below: 


$$
L_{i j q}\left(\beta_{i}\right)=\exp \left(\beta_{i} x_{i j q}\right) /\left(\Sigma_{k} \exp \left(\beta_{i} x_{i k q}\right)\right.
$$

The unconditional probability is then calculated by integrating the expression above over all possible values of $\beta_{i}$ :

$$
P_{i j q}=\int L_{i j q}\left(\beta_{i}\right) f\left(\beta_{i}\right) d \beta_{i}
$$

The probability, conditional on $\beta_{i}$, of a given sequence of choices $\mathrm{y}_{i}$ made by participant $i$ across all questions is as below, where $y(i, q)$ is the option chosen in question $q$.

$$
L_{i, y i}\left(\beta_{i}\right)=\prod_{q} L_{i, y(i, q), q}\left(\beta_{i}\right)
$$

The unconditional probability for this sequence of choices is finally

$$
P_{i, y i}=\int L_{i, y i}\left(\beta_{i}\right) f\left(\beta_{i}\right) d \beta_{i}
$$

The population-level parameters $\theta$ (mean and standard deviation) can be estimated using maximum simulated likelihood. The individual-level coefficients $\beta_{i}$ can then be derived from conditioning the observed sequence of choices $y_{i}$ on the estimated population-level parameters (Revelt and Train 2000). Using Bayes' rule, the conditional density of $\beta_{i}$ is expressed as below, where $x_{i}$ represents $x_{i j q}$ for all options $j$ and questions $q$.

$$
h\left(\beta_{i} \mid y_{i}, x_{i}, \theta\right)=P\left(y_{i} \mid x_{i}, \beta_{i}\right) f\left(\beta_{i} \mid \theta\right) / P\left(y_{i} \mid x_{i}, \theta\right)
$$

The conditional mean of $\beta_{i}$ is then the integral of the conditional density over all values of $\beta_{i}$ (expression 7), which can be approximated by simulation.

$$
\overline{\beta_{i}}=\int \beta_{i} h\left(\beta_{i} \mid y_{i}, x_{i}, \theta\right) d \beta_{\mathrm{i}}
$$

The model was specified as in expression 8 below, where the utility for participant $i$ of an option $j$ in a given question depends on:

- the additional walking time, if the option was Option B (WalkTime $)_{j}$.

- a dummy variable $A_{j}$ representing Option A, which measures the utility of crossing the best possible road, i.e. a road with 1 lane, wide central reservation, low traffic density, and 10mph speed.

- dummy variables for road conditions in Option A other than the best possible ones: 2 or 3 lanes (Lanes $2_{j}$, Lanes $3_{j}$ ), narrow or no central reservation (CRnarrow ${ }_{j}$, CRno ${ }_{j}$ ),

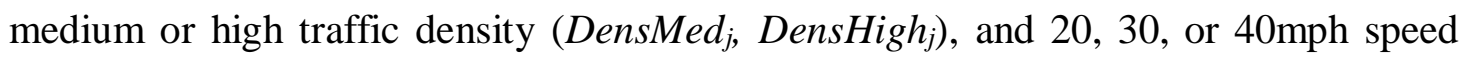
$\left(\right.$ Speed20 ${ }_{j}$, Speed30 ${ }_{j}$, Speed $\left.40_{j}\right)$.

- a dummy variable $C_{j}$ representing Option C.

- interactions between options $\mathrm{A}$ and $\mathrm{C}$ and a dummy variable representing dark scenarios $\left(A_{j}\right.$ Dark $_{j}, C_{j}$ Dark $\left._{j}\right)$. 


$$
\begin{aligned}
U_{i, j} & =\beta_{1} * \text { WalkTime }_{j}+\beta_{2} * A_{j}+\beta_{3} * \text { Lanes }_{j}+\beta_{4} * \text { Lanes }_{j}+\beta_{5} * \text { CRnarrow }_{j}+\beta_{6} * \text { CRno }_{j}+ \\
& +\beta_{7} * \text { DensMed }_{j}+\beta_{8} * \text { DensHigh } \\
& +\beta_{9} * \text { Speed } 20_{j}+\beta_{10} * \text { Speed }_{3}+\beta_{j}+\beta_{11} * \text { Speed }_{4} O_{j}+ \\
& +\beta_{12} * C_{j}+\beta_{13} * A_{j} \text { Dark }_{j}+\beta_{14} * C_{j} \text { Dark }_{j}+\varepsilon_{i, j}
\end{aligned}
$$

The option implicit in the case when all variables are equal to 0 is Option B (cross in a pedestrian platform) during daytime and without any extra walking. All coefficients were assumed to be random with normal distributions.

Two models were estimated. Model 1 uses the whole sample. Model 2 uses data for the group of participants in the Hereford case study who stated in a preliminary question that they make actual walking trips across the road. This model is used in Subsection 5.3 to compare the results of the stated and revealed preference exercises. Data for Option C (Do not make the trip) was not included in this model in order for the model to be comparable with the revealed preference model, which only includes data on actual trips.

\section{Results}

Table 2 shows the estimated population-level coefficients of the SP1 model. As an example of the interpretation of the coefficients, in the model for the whole sample, an increase of 1 minute in walking time leads to a decrease of -0.37 in the log-odds of a participant choosing a given option. A scenario with a road with 3 lanes, rather than 1 lane, leads to a decrease of 8.10 in the log-odds. 
Table 2: SP1 models

\begin{tabular}{|c|c|c|c|c|c|c|}
\hline & \multicolumn{2}{|c|}{ Model 1} & \multicolumn{4}{|c|}{ Model 2} \\
\hline & \multicolumn{2}{|c|}{ Whole sample } & \multicolumn{4}{|c|}{ For RP Comparison } \\
\hline & Coefficient & $\mathbf{z}$ & & Coefficient & $\mathbf{z}$ & \\
\hline \multicolumn{7}{|l|}{ Mean } \\
\hline Option A (Cross informally) & - & & & - & & \\
\hline Option C (Do not make trip) & -9.67 & -18.6 & $* * *$ & N/A & & \\
\hline Walking time (minutes) & -0.37 & -13.9 & **** & -0.25 & -7.2 & **** \\
\hline Lanes $=2$ & -3.52 & -12.0 & **** & -1.81 & -6.4 & **** \\
\hline Lanes $=3$ & -8.10 & -14.6 & $* * *$ & -5.35 & -8.3 & $* * *$ \\
\hline Central reservation $=$ narrow & -0.98 & -3.2 & **** & - & & \\
\hline Central reservation $=$ no & -2.13 & -7.4 & $* * *$ & -0.86 & -3.8 & $* * *$ \\
\hline Traffic density $=$ medium & -3.61 & -11.8 & $* * *$ & -1.60 & -5.9 & **** \\
\hline Traffic density=high & -5.43 & -14.4 & *** & -2.13 & -6.5 & *** \\
\hline Speed $=20 \mathrm{mph}$ & - & & & - & & \\
\hline Speed $=30 \mathrm{mph}$ & -0.47 & -2.0 & * & - & & \\
\hline Speed $=40 \mathrm{mph}$ & -3.05 & -4.6 & $* * *$ & N/A & & \\
\hline Option A * Dark & -2.11 & -7.3 & $* * *$ & -0.77 & -2.7 & **** \\
\hline Option C * Dark & 1.09 & 4.1 & **** & N/A & & \\
\hline
\end{tabular}

Standard deviation

Option A (Cross informally)

\begin{tabular}{|c|c|c|c|c|c|c|}
\hline Option C (Do not make trip) & 2.83 & 8.5 & **** & N/A & & \\
\hline Walking time (minutes) & 0.31 & 11.5 & **** & 0.22 & 7.6 & **** \\
\hline Lanes $=2$ & 1.87 & 4.9 & **** & 1.44 & 3.8 & **** \\
\hline Lanes $=3$ & 3.65 & 7.7 & $* * *$ & 3.13 & 4.9 & $* * *$ \\
\hline Central reservation $=$ narrow & 3.47 & 7.2 & **** & & & \\
\hline Central reservation $=$ no & 2.53 & 6.6 & $* * *$ & 0.78 & 1.9 & * \\
\hline Traffic density $=$ medium & 2.74 & 7.8 & **** & 1.38 & 4.0 & **** \\
\hline Traffic density=high & 2.71 & 6.6 & $* * *$ & 0.87 & 1.6 & \\
\hline
\end{tabular}

Speed $=20 \mathrm{mph}$

Speed $=30 \mathrm{mph} \quad 1.82 \quad 3.8^{* * *}$

Speed $=40 \mathrm{mph}$

Option A * Dark 2.01

Option C* Dark 1.89

Number of observations 15000

Number of participants $\quad 500$

Initial log-likelihood $\quad-5493$

Final log-likelihood $\quad-2724$

$3.9{ }^{* * *} \quad \mathrm{~N} / \mathrm{A}$

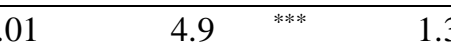

$1.89 \quad 5.1 \quad{ }^{* * *} \quad$ N/A

N/A

$2.4^{* *}$

$\rho 2$

0.50

3186

$\begin{array}{rr}3186 \\ 500 & 181\end{array}$

Notes: Model: Mixed Logit. $z$ : z ratio. Significance levels: ${ }^{* * *} 1 \%,{ }^{* *} 5 \%,{ }^{*} 10 \%$. N/A: Not applicable. "-": Variable was not included in the final model because it was not significant at the $10 \%$ level in preliminary models. Model 2 (For RP comparison): SP1 model to compare with revealed preference model (Subsection 5.3 of the paper), including only Hereford participants who currently cross the road.

In the model for the whole sample (Model 1), the coefficients of walking time and Option C were negative, which shows that participants prefer shorter walking times, and to make, rather than not to make the trip. The coefficient of Option A was not significant at the $10 \%$ level, i.e. given the survey design and sample size, we found no statistical evidence that participants prefer crossing a road with the best possible characteristics in a location with no crossing facilities (Option A) rather than crossing in a location where the road goes through a tunnel (Option B), when both alternatives imply no detour (i.e. when walking time=0). Both 
alternatives yield 0 utility. The $20 \mathrm{mph}$ speed coefficient was not significant, i.e. there is no statistical evidence of a preference towards crossing a road with $20 \mathrm{mph}$ speed, compared with one with 10mph speed. The coefficients of all other road attributes were negative, which shows that participants prefer to avoid crossing a road with 2 or 3 lanes, a narrow central reservation or no central reservation, medium or high traffic density, and 30, or $40 \mathrm{mph}$ speed, compared with a road with 1 lane, a wide central reservation, low traffic density, and 10mph speed. The relative magnitudes of the 2 vs. 3-lane, narrow vs. no central reservation, medium vs. high density, and 30mph vs. $40 \mathrm{mph}$ speed variables were as expected: worse conditions yield less utility.

The interaction between the 'dark' dummy variable and Option $\mathrm{C}$ is positive and the interaction with Option A is negative. This suggests that, after dark, participants have a stronger propensity for not making the trip (Option C) and prefer to cross in a location where the road goes through a tunnel (Option B) rather than crossing the best possible road in a location without crossing facilities (Option A), when both alternatives imply no detour. The standard deviations of the coefficients were all significant, confirming that there is heterogeneity in the preferences.

Most of the results above also apply to Model 2. The main difference is that in this model the 30mph speed coefficient was not significant.

\subsection{SP2: informal crossing vs. monetary benefits or costs}

\section{Design}

The second stated preference exercise (SP2) estimated the participants' willingness to pay to avoid crossing a road with in a location with no designated crossing facilities. The exercise was customized for three trip purposes: work, shopping, and leisure. Leisure trips had three types of possible destinations: (1) restaurant/pub/café; (2) park, playing field, or community/leisure centre; and (3) house of someone known to the participant (friend, neighbour, or relative). Each question showed two locations, one on each side of the road:

- For work trips, there were two locations for the same job, each with a different salary.

- For shopping trips and leisure trips to restaurants/pubs/cafés, the two locations offered the same products but with different prices.

- For leisure trips to parks, playing fields, or community/leisure centres, the two locations had the same characteristics but a voucher would be given in one of them. 
- For leisure trips to the house of someone, it was assumed that the trip was made by bus and two alternative bus stops were available, corresponding to different charging zones, so the fare would be different.

Each question contained two options (cross/do not cross), but they were presented in two different versions, to test for differences in responses when the trade-off scenario was defined in terms of benefits and in terms of costs.

In Version 1, the participant was financially better off by choosing to cross the road. Option A was to cross a road with specified design and traffic characteristics in a location with no crossing facilities and receive a higher salary; pay a cheaper shopping or restaurant/pub/café bill; receive a voucher; or pay a cheaper bus fare on the other side, compared with the current situation. Option B was not to cross the road and receive the same salary; pay the current bill; do not receive a voucher; or pay the current bus fare on the same side of the road. The left side of Figure 4 is an example of a Version 1 scenario for shopping trips.

In Version 2, shown only for shopping trips and leisure trips to restaurants/pubs/cafés or to the house of someone, the participant was financially worse off by choosing not to cross the road. Option A was to cross the road in a location with no crossing facilities and pay the same shopping bill, restaurant/pub/café bill, or bus fare as now. Option B was not to cross the road and pay a higher bill or bus fare on the same side of the road. The right side of Figure 4 is an example of a Version 2 scenario for shopping trips.

Figure 4: SP2 - example of questions

Version 1
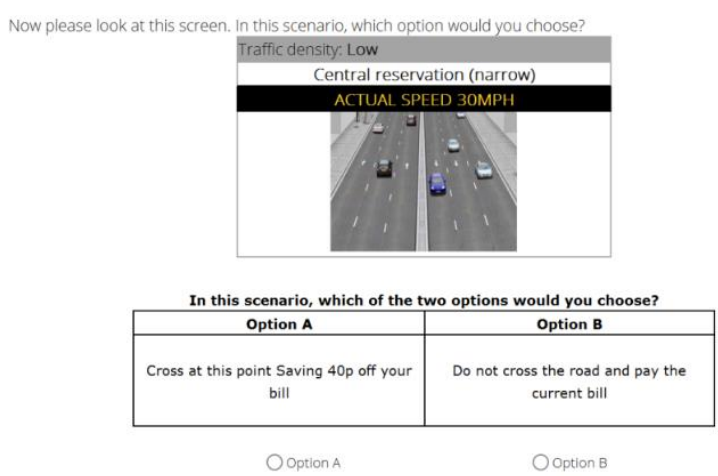

Version 2

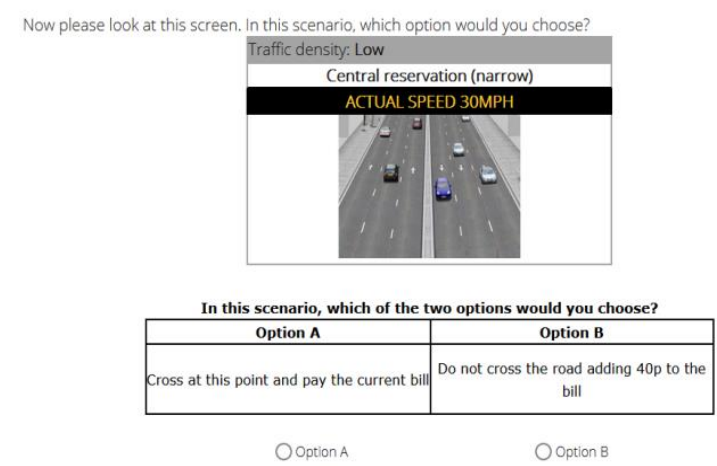

The attributes of the problem were the monetary value in Option A (ranging from $£ 0.40$ to $£ 4$, in increments of $£ 0.40$ ) and the characteristics of the road in Option A (with the same range of values as in SP1). Again, a constraint was applied so that scenarios with high traffic density did not have the highest speed value. It was implicit that it is necessary to cross the road twice to go to the location on the other side and return home. The only exception was for 
trips to the house of someone, which were assumed to be made by bus, and so required crossing the road only once (as the bus stop would be on the 'home' side in one direction). In this case, the range of monetary values shown was halved. The exercise consisted of eight questions.

As in the SP1 exercise, participants were identified as having a potential trip purpose based on whether they had indicated that the places associated with that purpose were within walking distance and that they currently go there (by any means of transport). In the case of leisure trips to the house of someone, the assessment considered whether bus stops were within walking distance and the participant currently goes there. Participants were then randomly allocated to one of the potential trip purposes and, in each question, to one of the two versions of the question.

As in the SP1 exercise, non-trading behaviour was identified in two preliminary choice questions, one showing the worst possible road in Option A and the minimum monetary value and another showing the best possible road in Option A and the maximum monetary value. The $3 \%$ of participants who chose Option $\mathrm{A}$ in the first question and the $15 \%$ who did not choose Option $\mathrm{A}$ in the second question were identified as non-traders and did not answered the exercise. Among the participants who answered the exercise, Option A was chosen in $33 \%$ of all questions.

\section{Model}

Choices were modelled using a mixed logit specification similar to the one used in the SP1 model (expressions 1-7 in Subsection 3.1). The utility $U_{i j}$ of option $j$ in a given question (expression 9) was assumed to depend on the monetary value (Money $y_{j}$ (a benefit in Option A or a cost in Option B), a dummy variable $A j$ representing Option A, and dummy variables representing road conditions other than the best possible ones. Option A was also interacted with the monetary value to account for the effect of benefits, compared to costs. The option implicit in the case when all variables are equal to 0 is Option B with no monetary value. All coefficients were assumed to be random with normal distributions. 


$$
\begin{aligned}
U_{i, j} & =\beta_{1} * \text { Money }_{j}+\beta_{2} * A_{j}+\beta_{3} * \text { Lanes }_{j}+\beta_{4} * \text { Lanes }_{j}+\beta_{5} * \text { CRnarrow }_{j}+\beta_{6} * \text { CRno }_{j}+ \\
& +\beta_{7} * \text { DensMed }_{j}+\beta_{8} * \text { DensHigh } \\
& +\beta_{9} * \text { Speed }_{2} O_{j}+\beta_{10} * \text { Speed }_{30}+\beta_{11} * \text { Speed }_{4} 0_{j}+ \\
& +\beta_{12} * \text { Money }_{j} * A_{j}+\varepsilon_{i, j}
\end{aligned}
$$

\section{Results}

Table 3 shows the estimated SP2 model coefficients. As an example of the interpretation of the coefficients, an increase of $£ 1$ in the monetary value (i.e. an increase in the benefit in Option A or a decrease in the cost in Option B) leads to an increase of 1.80 in the log-odds of

\begin{tabular}{|c|c|c|c|}
\hline & Coefficient & $\mathbf{z}$ & \\
\hline \multicolumn{4}{|l|}{ Mean } \\
\hline Option A (Cross informally) & - & & \\
\hline Monetary value $(£)$ & 1.80 & 10.1 & $* * *$ \\
\hline Lanes $=2$ & -1.77 & -10.3 & $* * *$ \\
\hline Lanes $=3$ & -4.49 & -12.7 & $* * *$ \\
\hline Central reservation $=$ narrow & - & & \\
\hline Central reservation $=$ no & -0.37 & -2.8 & $* * *$ \\
\hline Traffic density $=$ medium & -1.49 & -10.0 & **** \\
\hline Traffic density=high & -3.23 & -10.3 & $* * *$ \\
\hline Speed $=20 \mathrm{mph}$ & - & & \\
\hline Speed $=30 \mathrm{mph}$ & - & & \\
\hline Speed $=40 \mathrm{mph}$ & -0.49 & -1.9 & $*$ \\
\hline Option A * Monetary value & - & & \\
\hline \multicolumn{4}{|l|}{ Standard deviation } \\
\hline Option A (Cross informally) & - & & \\
\hline Monetary value $(£)$ & 2.42 & 11.6 & $* * *$ \\
\hline Lanes $=2$ & 1.43 & 4.7 & $* * *$ \\
\hline Lanes $=3$ & 2.47 & 5.9 & $* * *$ \\
\hline Central reservation $=$ narrow & - & & \\
\hline Central reservation $=$ no & 0.39 & 1.3 & \\
\hline Traffic density $=$ medium & 1.10 & 3.7 & $* * *$ \\
\hline Traffic density $=$ high & 1.77 & 4.8 & $* * *$ \\
\hline Speed $=20 \mathrm{mph}$ & - & & \\
\hline \multicolumn{4}{|l|}{ Speed $=30 \mathrm{mph}$} \\
\hline Speed $=40 \mathrm{mph}$ & 1.56 & 4.1 & $* * *$ \\
\hline Option A * Monetary value & - & & \\
\hline Number of observations & \multicolumn{3}{|c|}{8528} \\
\hline Number of participants & \multicolumn{3}{|c|}{533} \\
\hline Initial log-likelihood & \multicolumn{3}{|c|}{-2956} \\
\hline Final log-likelihood & \multicolumn{3}{|c|}{-1795} \\
\hline$\rho 2$ & \multicolumn{3}{|c|}{0.39} \\
\hline \multicolumn{4}{|c|}{$\begin{array}{l}\text { Notes: Model: Mixed Logit. } z: \mathrm{z} \text { ratio. Significance levels: }{ }^{* * *} 1 \%,{ }^{* *} 5 \%,{ }^{*} 10 \% . \text { N/A: Not } \\
\text { applicable. "-": Variable was not included in the final model because it was not significant at } \\
\text { the } 10 \% \text { level in preliminary models. }\end{array}$} \\
\hline
\end{tabular}
a participant choosing a given option.

\section{Table 3: SP2 model}

The coefficient of the monetary value was positive, which means that participants prefer higher benefits or lower costs, as expected. The interaction of the monetary value and Option 
A was not significant, which means that the design of the exercise (i.e. offering a benefit vs. a cost) did not influence the participants' sensitivity to the monetary value. The $20 \mathrm{mph}$ and $30 \mathrm{mphtraffic}$ speed coefficients were not significant at the $10 \%$ level, i.e. given the survey design and sample size, there is no statistical evidence that participants prefer crossing roads with traffic speeds of $10 \mathrm{mph}$, compared with $20 \mathrm{mph}$ or $30 \mathrm{mph}$. The coefficient of the narrow central reservation was also not significant. The sign and magnitude of the coefficients of the other road attributes were as expected: worse conditions for pedestrians yield less utility. The coefficient of Option A was not significant. The standard deviations of the coefficients were significant, with the exception of the central reservation variable.

\subsection{SP3: formal crossing vs. walking time}

\section{Design}

The third stated preference exercise estimated trade-offs between walking time and crossing the road using a certain type of pedestrian crossing facility. Each question had four options (see example in Figure 5):

- Options A and B: Walk a specified number of extra minutes in order to cross the road using a given type of crossing facility

- Option C: Walk a specified number of extra minutes in order to cross in a pedestrian platform, i.e. a location where the road goes through a tunnel

- Option D: Do not make the trip

Figure 5: SP3 - example of question

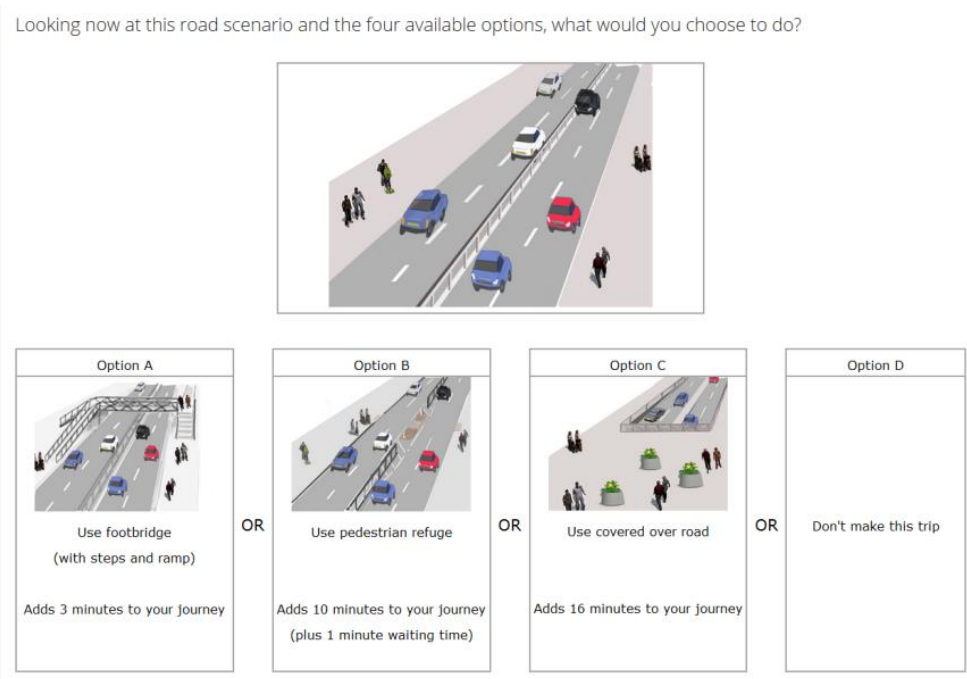

The attributes of the problem were the additional walking times in Options $\mathrm{A}, \mathrm{B}$, and $\mathrm{C}$ (from 2 to 20 minutes, in increments of 2 minutes) and the types of crossing facilities offered 
in Options $\mathrm{A}$ and $\mathrm{B}$ (pedestrian refuge, straight signalised crossing, staggered signalised crossing, footbridge, high-quality footbridge, or underpass). The high-quality footbridge was only shown to participants in the Hull case study area. A constraint were applied to the experimental design so that the additional walking time presented in Option $\mathrm{C}$ was always longer than the walking times presented in Options A and B. Waiting times to cross the road at pedestrian refuges and straight and staggered signalised crossings were not included as an attribute with different levels in the design. However, these times were shown in the questions, and were the same as those indicated by each participant in a preliminary question.

The exercise consisted of eight questions. The text introducing the exercise was customized so that participants knew the purpose of the walking trip, which was the same as in the SP1 exercise. Across all participants, Options A or B were chosen in $82 \%$ of all questions, Option $\mathrm{C}$ in $16 \%$ and Option $\mathrm{C}$ in $2 \%$.

\section{Model}

Choices were modelled using a mixed logit specification similar to the one used in the SP1 and SP2 models. The utility $U_{i j}$ of option $j$ in a given question for participant $i$ (expression 10) was assumed to depend on the additional walking time (WalkTime f $_{j}$ ) in Options A, B, or C, the waiting time (WaitTime ) $_{\text {) }}$ in Options A or B (which applies only to pedestrian refuges and straight and staggered signalised crossings), dummy variables representing the possible types of crossing facilities presented in Options A or B, and a dummy $D_{j}$ for Option D.

$$
\begin{aligned}
& U_{i, j}=\beta_{1} * \text { WalkTime }_{j}+\beta_{2} * \text { WaitTime }_{j}+\beta_{3} * \text { Refuge }_{j}+\beta_{4} * \text { Straight }_{j}+\beta_{5} * \text { Staggered }_{j}+ \\
& +\beta_{6} * \text { Bridge }_{j}+\beta_{7} * H Q B \text { Bidge }{ }_{j}+\beta_{8} * \text { Underpass }_{j}+\beta_{9} * D_{j}
\end{aligned}
$$

The option implicit in the case when all the variables are equal to 0 is Option $\mathrm{C}$ with 0 walking time, i.e. cross in a location where the road goes through a tunnel, without any extra walking. All coefficients were assumed to be random with normal distributions.

As in the SP1 analysis, two SP3 models were estimated. Model 1 uses the whole sample. Model 2 uses data for the group of Hereford who make actual walking trips across the road. This model is used in Subsection 5.3 to compare the results of the stated and revealed preference exercises. Data for Option D (Do not make the trip) was not included in this model in order for the model to be comparable with the revealed preference model, which only includes data on actual trips. 


\section{Results}

Table 4 shows the estimated SP3 model coefficients. As an example of the interpretation of the coefficients, in the model for the whole sample, an increase of 1 minute in walking time leads to a decrease of -0.65 in the log-odds of a participant choosing a given option in the SP3 exercise.

Table 4: SP3 models

\begin{tabular}{|c|c|c|c|c|c|c|}
\hline & \multicolumn{3}{|c|}{$\begin{array}{c}\text { Model } 1 \\
\text { All }\end{array}$} & \multicolumn{3}{|c|}{$\begin{array}{c}\text { Model } 2 \\
\text { For RP comparison }\end{array}$} \\
\hline & Coefficient & $\mathbf{z}$ & & Coefficient & $\mathbf{z}$ & \\
\hline \multicolumn{7}{|l|}{ Mean } \\
\hline Option D (Do not make trip) & -19.73 & -14.8 & $* * *$ & N/A & & \\
\hline Walking time (minutes) & -0.65 & -18.1 & $* * *$ & -0.78 & -10.9 & $* * *$ \\
\hline Waiting time (minutes) & -0.89 & -4.0 & $* * *$ & - & & \\
\hline Pedestrian refuge & -2.62 & -7.4 & $* * *$ & -2.30 & -6.7 & $* * * *$ \\
\hline Straight signalised crossing & 1.08 & 4.1 & $* * *$ & - & & \\
\hline Staggered signalised crossing & 1.64 & 4.4 & $* * *$ & - & & \\
\hline Footbridge & -1.72 & -7.3 & $* * *$ & -2.13 & -5.6 & $* * *$ \\
\hline High-quality footbridge & - & & & N/A & & \\
\hline Underpass & -4.59 & -11.5 & **** & -4.43 & -6.5 & $* * *$ \\
\hline \multicolumn{7}{|l|}{ Standard deviation } \\
\hline Option D (Do not make trip) & 7.77 & 11.7 & $* * *$ & & & \\
\hline Walking time (minutes) & 0.52 & 16.6 & *** & 0.59 & 9.5 & $* * *$ \\
\hline Waiting time (minutes) & 1.54 & 9.8 & $* * *$ & - & & \\
\hline Pedestrian refuge & 3.82 & 12.3 & $* * *$ & 3.07 & 7.2 & **** \\
\hline Straight signalised crossing & 1.72 & 6.9 & $* * *$ & - & & \\
\hline Staggered signalised crossing & 1.08 & 3.2 & $* * *$ & - & & \\
\hline Footbridge & 3.80 & 12.8 & $* * *$ & 3.76 & 8.5 & $* * *$ \\
\hline High-quality footbridge & - & & & N/A & & \\
\hline Underpass & 5.85 & 11.8 & $* * *$ & 5.41 & 7.5 & *** \\
\hline Number of observations & \multicolumn{3}{|c|}{20888} & \multicolumn{3}{|c|}{5100} \\
\hline Number of participants & \multicolumn{3}{|c|}{653} & \multicolumn{3}{|c|}{218} \\
\hline Initial log-likelihood & \multicolumn{3}{|c|}{-7239} & \multicolumn{3}{|c|}{-1868} \\
\hline Final log-likelihood & \multicolumn{3}{|c|}{-3482} & \multicolumn{3}{|c|}{-979} \\
\hline$\rho 2$ & \multicolumn{3}{|c|}{0.52} & \multicolumn{3}{|c|}{0.48} \\
\hline \multicolumn{7}{|c|}{$\begin{array}{l}\text { Notes: Model: Mixed Logit. } z: \mathrm{z} \text { ratio. Significance levels: }{ }^{* * *} 1 \%,{ }^{* *} 5 \%,{ }^{*} 10 \% . \text { N/A: Not applicable. "-" Variable } \\
\text { was not included in the final model because it was not significant at the } 10 \% \text { level in preliminary models. Model } \\
2 \text { (For RP comparison): SP3 model to compare with revealed preference model (Subsection } 5.3 \text { of the paper). } \\
\text { Model } 2 \text { includes only Hereford participants who currently cross the road. }\end{array}$} \\
\hline
\end{tabular}

In the model using the whole sample (Model 1), the coefficients of walking time, waiting time, and Option D were negative, i.e. participants prefer shorter, rather than longer walking and waiting times, and to make, rather than not to make the trip. The coefficient of waiting time was more negative than the walking time coefficient, which suggests that participants are more sensitive to waiting time than to walking time.

The coefficients of the underpass, pedestrian refuge, and footbridge, were negative, i.e. participants prefer to avoid using these crossing facilities, compared with the omitted 
alternative (a location where the road goes through a tunnel). The coefficient of the highquality footbridge was not significant at the $10 \%$ level. The coefficients of the straight and staggered signalised crossings were positive. This means that if there is no waiting time, participants prefer to use these crossing facilities rather than cross in a location where the road goes through a tunnel. However, for waiting times above 73 seconds and 110 seconds, respectively, the utility of straight and staggered signalised crossings (given by the sum of the coefficients of these variables and the product of waiting time and the coefficient of waiting time) becomes negative. In this case, those two types of facilities are less preferred than crossing in a location where the road goes through a tunnel (which, as the omitted alternative, has 0 utility). Underpasses are the least preferred facilities, followed by pedestrian refuges (with no waiting time), and footbridges. Pedestrian refuges with a waiting time of around 2 minutes and 13 seconds yield the same utility as an underpass. The standard deviations of the coefficients were all significant.

Most of these results also apply to Model 2. However, in Model 2, the coefficients of the straight and staggered signalised crossings were not significant.

\section{Revealed preference exercise}

\section{Design}

The revealed preference exercise estimated trade-offs between walking time and the characteristics of the road and crossing facilities in the locations where participants currently cross the road in the real world. Participants were shown a map of a wide area around the case study road and asked to indicate the time of day (daytime or night-time) and the route of a typical walking trip from home for each of three trip purposes (work, shopping, and leisure). The route was defined by five points: 1) where the trip started (i.e. home), 2) where the route joined the road, 3) where the route crossed the road, 4) where the route left the road, and 5) where the trip ended. The question was asked only to participants who had stated that there are places associated with that trip purpose on the other side of the road and that they usually walk there.

The analysis used data from the participants in the Hereford case study only. Robust revealed preference models of choices of crossing points could not be estimated in the Hull case study, because, due to the presence of physical barriers along most of the road length, participants crossed in a very small number of points. In Hereford, trips where the crossing point location indicated by the participant on the interactive map was more than $50 \mathrm{~m}$ distant 
from the road were also excluded. The resulting dataset included information for 291 walking trips (18 for work, 132 for shopping, and 141 for leisure), made by 189 participants. The five points indicated by the participants for each trip were converted into walking routes, by estimating the shortest path between points, using a street network model within a geographic information system.

A choice set of possible walking routes was then created for each trip of each participant. This choice set included all combinations of routes from home to all possible crossing points and from these points to the trip destination. Crossing points included all crossing facilities as well as informal crossing points. The latter were defined as points where there are no crossing facilities but where crossing was both possible (because there were no physical barriers) and likely (because the point was along pedestrian desire lines, i.e. at junctions with side roads or near bus stops or relevant trip destinations). For each trip, the defined choice set of possible crossing points included the crossing point this is part of the fastest route and the crossing points that are part of all routes where the excess walking time was less than 20 minutes, compared with the fastest route. The walking speed assumed in all links of the pedestrian network was $5 \mathrm{~km} / \mathrm{h}$.

A dataset was then created with a record for each of the possible crossing points for each trip of each participant. This dataset was joined with data with the characteristics of the crossing point, i.e. type of crossing facility (pedestrian refuge, straight signalised, staggered signalised, underpass, or no facility), waiting time (at pedestrian refuges and straight or signalised crossings), and, in the case of informal crossing points, also the characteristics of the road (number of lanes in each direction, central reservation, and traffic volume). All this data, except traffic volume, was collected in site audits. The central reservation attribute was coded with only two values (yes $v s$. no); due to the variety of different widths encountered, any classification into "narrow" and "wide" would be arbitrary. Traffic volume was used instead of traffic density, as available data, from the UK Department of Transport, provides only traffic volume. This variable was coded with two values: high vs. not high. The cut-off point was 40,000 vehicles/day, a value that separates two clusters of road sections, one with volumes between 17,652 and 23,523 and another with volumes between 43,850 and 44,856. Traffic speed was not considered due to the lack of data. However, as all the speed variables in the Hereford stated preference model (Model 2 in Table 2) were statistically insignificant, this simplification does not impact on the comparison between the stated preference and revealed preference models. 


\section{Model}

Choices were modelled using a mixed logit specification similar to the one used in the SP models. The utility $U_{i j}$ of crossing point $j$ for a trip with a given purpose made by participant $i$ (expression 11) was assumed to depend on the additional walking time (i.e. the detour in relation to the fastest route, expressed in minutes), waiting time at crossing facilities, a dummy variable (Informal $)_{j}$ ) representing all informal crossing points, dummy variables representing road conditions other than the best ones at informal crossings (i.e. 2 or 3 lanes, no central reservation, and high traffic volume), and dummy variables representing types of crossing facilities (pedestrian refuges, staggered signalised crossings, and underpasses). The informal crossings dummy was also interacted with a dummy variable representing trips made after dark (Informal Dark $_{j}$ ). The omitted alternative, with utility 0, is to use the fastest route and a straight signalised crossing with no waiting time. All coefficients were assumed to be random with normal distributions.

$$
\begin{aligned}
U_{i, j} & =\beta_{1} * \text { WalkTime }_{j}+\beta_{2} * \text { WaitTime }_{j}+\beta_{3} * \text { Informal }_{j}+\beta_{4} * \text { Lanes }_{j}+\beta_{5} * \text { Lanes }_{j}+\beta_{6} * \text { CRno }_{j}+ \\
& +\beta_{7} * \text { VolumeHigh }_{j}+\beta_{8} * \text { Refuge }_{j}+\beta_{9} * \text { Staggered }_{j}+\beta_{10} * \text { Underpass }_{j}+\beta_{11} * \text { Informal }_{j} \text { Dark }_{j}+\varepsilon_{i, j}
\end{aligned}
$$

\section{Results}

Table 5 shows the estimated revealed preference model and its comparison with the stated preference models. Column (1) shows the revealed preference model coefficients. As an example of the interpretation of the coefficients, an increase of 1 minute walking time leads to a decrease of -0.77 in the log-odds of a participant choosing a given crossing point for a walking trip across the road. 
Table 5: Revealed preference model and comparison with stated preference models

\begin{tabular}{ccccccc}
\hline & \multicolumn{4}{c}{ RP Model } & \multicolumn{4}{c}{ Comparison RP-SP models } \\
\hline$(1)$ & $(2)$ & $(3)$ & $(4)$ & $(5)$ & $(6)$ \\
Coeff. & $\mathbf{z}$ & Marginal & WTW & WTW & Ratio WTW \\
& & WTW (RP) & (RP) & (Hereford SP) & RP/SP \\
\hline
\end{tabular}

\section{Mean}

\begin{tabular}{|c|c|c|c|c|c|c|}
\hline Walking time (minutes) & -0.77 & $-8.1^{* * *}$ & & & & \\
\hline Waiting time (minutes) & & & 0.00 & & & \\
\hline Informal crossings & -1.99 & $-4.0^{* * *}$ & 2.87 & & & \\
\hline Lanes $=2$ & -0.95 & $-2.2^{* *}$ & 1.38 & 4.25 & 6.85 & 0.62 \\
\hline Lanes $=3$ & -1.23 & $-2.0^{* *}$ & 1.78 & 4.65 & 20.90 & 0.22 \\
\hline Central reservation $=$ no & & & 0.00 & 2.87 & 3.24 & 0.89 \\
\hline Traffic volume $=$ high & -1.51 & $-2.0^{* *}$ & 2.23 & 5.10 & $8.08^{\#}$ & 0.63 \\
\hline Pedestrian refuge & -1.22 & $-4.1^{* * * *}$ & 1.79 & 1.79 & 3.36 & 0.53 \\
\hline Staggered signalised crossing & & & 0.00 & 0.00 & - & - \\
\hline Underpass & & & 0.00 & 0.00 & - & - \\
\hline Informal crossing $*$ Dark & & & 0.00 & 0.00 & - & - \\
\hline
\end{tabular}

\section{Standard Deviation}

\begin{tabular}{lrll} 
Walking time (minutes) & 0.33 & $4.4^{* * *}$ \\
\hline Waiting time (minutes) & - & \\
\hline Informal crossings & 1.37 & $2.4^{* *}$ \\
\hline Lanes $=2$ & 0.08 & 0.1 \\
Lanes $=3$ & 0.20 & 0.1 \\
Central reservation=no & - & \\
Traffic volume=high & 1.64 & $1.6^{*}$ \\
\hline Pedestrian refuge & 1.04 & $2.1^{* *}$ \\
Staggered signalised crossing & - & - \\
Underpass & - & \\
\hline Informal crossing * Dark & - & 189 \\
\hline Number of observations & -957 \\
Number of participants & -547 \\
Initial log-likelihood & 0.43 \\
Final log-likelihood & $\rho 2$ &
\end{tabular}

Notes: Model: Mixed Logit. RP: revealed preference. SP: stated preference; Coeff.: coefficient. $z: \mathrm{z}$ ratio. WTW: willingness to walk (minutes). Significance levels: ${ }^{* * *} 1 \%,{ }^{* * *} 5 \%,{ }^{*} 10 \%$. "-" Variable was not included in the final model because it was not significant at the $10 \%$ level in preliminary models. " Value refers to traffic density.

The walking time coefficient was negative, as expected. The waiting time coefficient was not significant, i.e., given the design of the revealed preference exercise, sample size, and the set of available variables to model the choices of crossing points, there is no statistical evidence that participants attach extra disutility to waiting time at pedestrian refuges or signalised crossings, compared with the general disutility of using those crossing facilities. The coefficient representing informal crossings was negative. This shows that participants prefer using a straight signalised crossing (the omitted alternative) rather than crossing in a location without crossing facilities. The coefficients of the road characteristics at informal crossing points were negative, as expected. This suggests that participants prefer to avoid 
crossing a road with 2 or 3 lanes, no central reservation, or high traffic volume, compared with a road with the best possible conditions, i.e. 1 lane, a central reservation, and not high traffic volume. In addition, the coefficient for 3 lanes was more negative than the one for 2 lanes. The coefficients of staggered signalised crossings and underpasses were not significant, i.e. there is no statistical evidence that participants prefer crossing using straight signalised crossings, rather than those two other types of crossing facilities. The coefficient of informal crossings for trips made after dark was not significant, i.e. there is no evidence that participants attach less utility to cross the road in locations without designated crossing facilities after dark, compared with daytime. The standard deviations of the traffic volume coefficients were not significant; all the other standard deviations were significant.

Column (3) of Table 5 shows the marginal willingness to walk to avoid crossing points with specific characteristics and use instead a straight signalised crossing with no waiting time. For example, when compared with a road with 1 lane, participants are willing to walk an additional 1.38 minutes to avoid crossing informally a road with 2 lanes and use a straight signalised crossing. The marginal willingness to walk was estimated as the mean, across all individuals, of the ratios between the individual-level coefficient of the variable representing each characteristic and the individual-level coefficient of walking time. This approach (which we also use in Section 5 to estimate a severance index and willingness to pay) is imperfect (as it is based on individual conditional distributions of the coefficients, not population distributions) but it has several advantages, as shown by Sillano and Ortúzar (2005). It allows for the segmentation of results (for example, by trip purpose) and avoids the shortcomings of other options: the ratio of population coefficients (which in our case are normally distributed) has an unstable distribution; fixing coefficients masks preference heterogeneity; and using log-normal distributions tends to inflate the estimates.

Column (4) adds, in the case of informal crossing points, the general marginal willingness to walk to avoid these points (2.87 minutes) to the marginal willingness to walk to avoid specific characteristics of the road. For example, participants are willing to walk an additional 4.25 minutes $(2.87+1.38)$ to avoid crossing informally a road with 2 lanes and use a straight signalised crossing instead.

Column (5) shows the values of the willingness to walk calculated from the SP1 and SP3 models that were estimated for the group of participants in Hereford who completed the revealed preference exercise (i.e. models 2 in Table 2 and Table 4). As above, the willingness to walk values were calculated as the mean, across all individuals, of the ratios between the 
individual-level coefficient of the variable representing each characteristic of the road or crossing facilities and the individual-level coefficient of walking time.

Finally, column (6) is the ratio between the willingness to walk values estimated from the revealed preference and stated preference exercises. The ratio is between 0.22 and 0.89 , depending on the variable. An overall ratio of 0.58 is used in Subsection 5.3 to scale the stated preference results. This is the average of the ratios obtained for the individual variables.

\section{Use of results in appraisal}

We combined the results of the stated and revealed preference exercises in an integrated approach to measure and value the barrier effect of roads. The approach is described in detail in the following five subsections, which are colour-coded. Figure 6 is an overview of the approach. The boxes in the figure use the same colour code as the subsections. Each box represents a quantity and includes, at the bottom, the number of the expression where that quantity is defined in the text.

The integrated approach consists of the following steps:

- Using the SP1 and SP3 results, calculate an index of the barrier effect and willingness to walk (WTW) to avoid roads with different characteristics and crossing facilities (Subsection $5.1 \square$ )

- Calculate the willingness to pay (WTP) associated with each value of the index, by relating the results of the three stated preference exercises (Subsection 5.2

- Scale willingness to walk and willingness to pay to the results of the revealed preference model (Subsection $5.3 \square$ ).

- Combine the index and willingness to pay values of roads and crossing facilities, considering walking time to the nearest crossing facility (Subsection 5.4 ).

- Adjust willingness to pay to account for the number of walking trips associated with each value of the index (Subsection 5.5 ). 
Figure 6: Approach to measure and value the barrier effect of roads

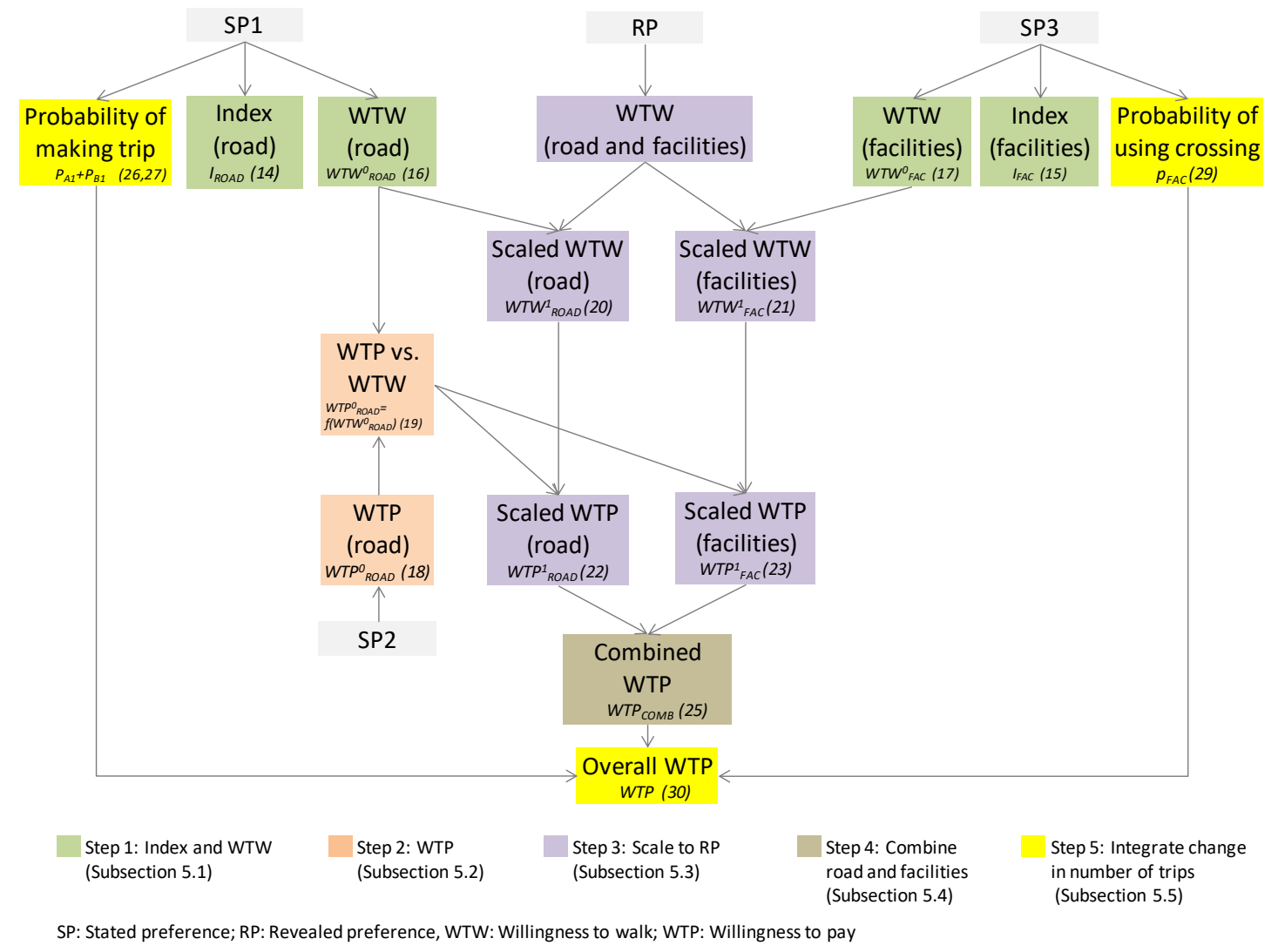

\subsection{Index of the barrier effect and willingness to walk}

The results of the SP1 and SP3 exercises were used to create an index of the barrier effect of roads with different characteristics and crossing facilities at a given point along the road. This index was defined as the disutility of crossing the road relative to the disutility of not making the trip and was calculated from the individual-level SP model coefficients. A ratio was derived for each individual by summing the individual-level coefficients representing the specified characteristics of roads and crossing facilities and then dividing by the coefficient of the "do not make the trip" option (Option C in the SP1 model or Option D in the SP3 model). The index is then the mean of the ratios across all individuals.

Expression 12 shows the index for roads $\left(I^{0}{ }_{R O A D}\right)$, where $\beta_{C l i}$ is the individual-level coefficient of Option $\mathrm{C}$ in the SP1 model for individual $i, \beta_{R O A D I i}$ is the sum of the coefficients representing the specified road conditions in the SP1 model, and $n$ is the sample size. Expression 13 shows the index for crossing facilities $\left(I_{F A C}^{0}\right)$, where $\beta_{D 3 i}$ is the individual-level coefficient of Option D in the SP3 model for individual $i, \beta_{F A C 3 i}$ is the coefficient of the specified facility, and $\beta_{\text {WaitTimesi }}$ is the coefficient of waiting time in the SP3 model. WaitTime is the specified waiting time at the facility. The two versions of the index are expressed in the 
same scale, as the option represented in the denominator ("do not make the trip") is the same in both versions.

$$
\begin{gathered}
I^{0_{R O A D}}=\sum_{i}\left(\beta_{R O A D I i} / \beta_{C I i}\right) / n \\
I^{0_{F A C}}=\sum_{i}\left(\left(\beta_{F A C 3 i}+\beta_{\text {WaitTime } 3 i} * \text { WaitTime }\right) / \beta_{D 3 i}\right) / n
\end{gathered}
$$

An index value of 0 means that the road or crossing facility yields the same disutility, on average, as the omitted option in the models (cross in a location where the road goes through a tunnel). We found that the road with the best possible characteristics for pedestrians (1 lane, wide central reservation, low traffic density, and 10mph speed) has an index of 0 . It should be noted that this value is not 0 by definition but because the estimated coefficient of Option $\mathrm{A}$ (which represents roads with the best possible conditions for pedestrians) was not statistically different from 0 in the SP1 model. A negative index means that the road or crossing facility has less disutility, on average, than a location where the road goes through a tunnel. We found this to be the case for straight and staggered signalised crossings with no waiting time (as they have a positive coefficient in the SP3 model). In these cases, we set the index to 0 . An index above 1 means that the road or crossing facility yields more disutility, on average, than not making the trip.

For analytical convenience, we then converted all the index values into a 0-100 scale. A value of 100 was assigned to the maximum value across the two indices (1.91). The other values were then scaled using the expressions below.

$$
\begin{gathered}
I_{R O A D}=100 * I^{0}{ }_{R O A D} / 1.91 \\
I_{F A C}=100 * I_{F A C}^{0} / 1.91
\end{gathered}
$$

Figure 7 shows some examples of the index of the barrier effect for some types of roads and crossing facilities. The upper table in Appendix 1 shows the index for all types of roads, i.e. all combinations of design and traffic characteristics. The road with an index of 100 has 3 lanes, no central reservation, medium traffic density, and $40 \mathrm{mph}$ traffic speed. The lower table in Appendix 1 shows the index for all types of crossing facilities, considering different waiting times. The crossing facility causing the highest barrier effect per se is the underpass (index=13), although pedestrian refuges with long waiting times (above 2 minutes) cause a stronger barrier effect. Straight and staggered signalised crossings only have a barrier effect above 0 when the waiting times are above 2 and 3 minutes, respectively.

The index can be disaggregated by trip purpose, using an approach similar as for the whole sample, considering the individual-coefficients of the sample participants who answered the 
stated preference exercises customized for that purpose. The disaggregated indices are shown in the tables in Appendix 2.

Figure 7: Index of the barrier effect: examples

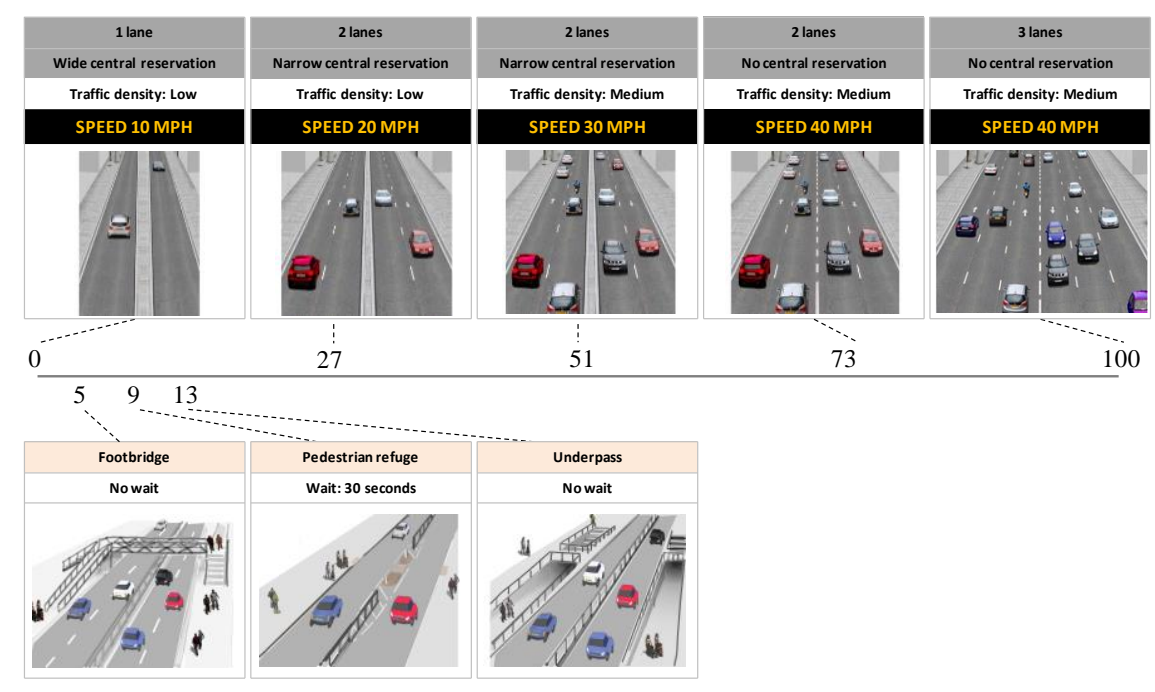

The willingness to walk to avoid crossing roads with specific characteristics $\left(W T W^{0}{ }_{R O A D}\right)$ and to avoid using specific crossing facilities $\left(W T W_{F A C}^{0}\right)$ was also calculated as the mean of individual-level ratios (shown in expressions 16 and 17). For each individual $i$, the ratio was derived by summing the individual-level coefficients representing the specified characteristics of roads and crossing facilities and then dividing by the SP1 and SP3 coefficients of walking

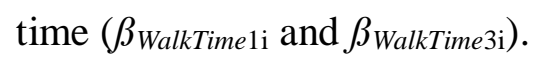

$$
\begin{gathered}
\text { WTW }{ }_{\text {ROAD }}=\sum_{i}\left(\beta_{\text {ROADIi }} / \beta_{\text {WalkTimeli } i}\right) / n \\
W_{T W^{0}}{ }_{\text {FAC }}=\sum_{i}\left(\left(\beta_{\text {FAC } 3 i}+\beta_{\text {WaitTime } 3 i} * \text { WaitTime }\right) / \beta_{\text {WalkTime } 3 i}\right) / n
\end{gathered}
$$

\subsection{Willingness to pay}

The willingness to pay to avoid crossing the road at a given location (expression 18) was then defined as the mean of the individual-level ratios between the sum of the individual-level SP2 coefficients representing the specified road conditions $\left(\beta_{R O A D 2 i}\right)$ and the SP2 coefficient of the monetary value $\left(\beta_{M O N E Y 2 i}\right)$ :

$$
W T P^{0}{ }_{R O A D}=\sum_{i}\left(\beta_{R O A D 2 i} / \beta_{M O N E Y 2 i}\right) / n
$$

We found a strong $\left(\mathrm{R}^{2}=0.94\right)$ linear relationship (expression 19) between willingness to walk and willingness to pay across all possible types of roads, i.e. all combinations of road attribute values. This strong relationship shows that the choices were consistent across the stated preference exercises, i.e. participants tended to have the same relative preferences about 
crossing different types of roads regardless of the type of trade-off involved (whether walking time or monetary values).

$$
W T P^{0}{ }_{R O A D}=0.1079 * W T W^{0}{ }_{R O A D}-0.1022
$$

\subsection{Scaling to revealed preferences}

In Section 4 of the paper we derived a scaling factor to adjust the willingness to walk values obtained from the stated preference exercise to values consistent with the participants' walking behaviour in the real world as calculated from the revealed preference exercise. That scaling factor, estimated as 0.58 , can be applied to the willingness to walk associated with the different types of roads and crossing facilities:

$$
\begin{gathered}
W T W^{l}{ }_{R O A D}=0.58^{*} W T W^{0}{ }_{R O A D} \\
W T W^{l}{ }_{F A C}=0.58^{*} W T W^{0}{ }_{F A C}
\end{gathered}
$$

The estimated relationship between willingness to pay and willingness to walk (expression 19) can then be applied not only to scale the willingness to walk associated with roads (expression 22) but also to derive the willingness to pay associated with crossing facilities (expression 23):

$$
\begin{gathered}
W T P^{l}{ }_{R O A D}=0.1079 * W T W^{l}{ }_{R O A D}-0.1022 \\
W T P^{l}{ }_{F A C}=0.1079 * W T W^{1}{ }_{F A C}-0.1022
\end{gathered}
$$

The tables in Appendix 1 show the willingness to pay (per trip) associated with the barrier effect of all types of roads and crossing facilities. The maximum value, corresponding to the road with an index of 100 , is $£ 2.67$. The maximum value for crossing facilities per se is $£ 0.29$ (for underpasses), although pedestrian refuges with long waiting times (above 3 minutes) have higher values. Appendix 2 shows the same type of information disaggregated by trip purpose. The values in the tables in the appendices are indicators of the current costs of the barrier effect (or the benefits of completely removing the effect). However, they can be used to calculate the benefits of policies for reducing the effect, as the difference between the willingness to pay associated with the road characteristics or crossing facilities in the prepolicy and post-policy scenarios.

\subsection{Combined assessment (characteristics of road and type and time to facilities)}

The analysis in the previous sections separated the assessment of the barrier effect of roads with specific characteristics and the effect of crossing facilities, at a given point along the 
road. However, the effect at that point also depends on the distance to the nearest crossing facility.

The index of the barrier effect at a point can be adjusted by combining the indices of roads and crossing facilities. We assumed that facilities located more than 10 minutes away do not mitigate the barrier effect felt at that point. This assumption was supported by the analysis of the probabilities derived from the SP1 model, which found that the probability of choosing Option B (walk to a crossing facility) with a detour of 20 minutes (i.e. go to the facility and come back to the same place but across the road) was the same as the probability of choosing Option A (cross the road in a location with no crossing facilities) with the worst possible road conditions. The assumption means that when the time to the nearest crossing facility is above 10 minutes, the index of the barrier effect depends only on the road conditions, and not on the presence of crossing facilities, and can therefore be set as the index of the road ( $I_{R O A D}$, previously defined in expression 14). When the walking time to the crossing facility is 0 minutes, the pedestrian is at that facility, so the index can be set as the index of the facility $\left(I_{F A C}\right.$, defined in expression 15).

We then assumed that for walking times between 0 and 10 minutes, the index varies proportionately in the $\left[I_{F A C}, I_{R O A D}\right]$ interval. The combined index $\left(I_{C O M B}\right)$ can therefore be expressed as in expression 24 below, where FacTime is the walking time to the nearest crossing facility. It was assumed that no adjustment is needed when $I_{R O A D}<I_{F A C}$, i.e. when the crossing facility causes a stronger barrier effect than crossing in a location without facilities. The combined willingness to pay $W_{T P} P_{\text {Сом }}$ can be calculated using expression 25 , which replacing indices with willingness to pay in expression 24.

$$
\begin{array}{rlr}
I_{C O M B}= & I_{F A C}+0.1 \text { FacTime }\left(I_{R O A D}-I_{F A C}\right) & \text { if } I_{R O A D} \geq I_{F A C} \\
& I_{R O A D} & \text { if } I_{R O A D}<I_{F A C} \\
W T P_{C O M B}= & & \\
& W T P^{l}{ }_{F A C}+0.1 \text { FacTime }\left(W T P^{l}{ }_{R O A D}-W T P^{l}{ }_{F A C}\right) & \text { if } I_{R O A D} \geq I_{F A C} \\
& W T P^{l}{ }_{R O A D} & \text { if } I_{R O A D}<I_{F A C}
\end{array}
$$

In practice, the adjustment means that the values of the index and willingness to pay shown in the upper table in Appendix 1 go down when taking into account the distance to crossing facilities. As an example, the index of the barrier effect of a road with 2 lanes, a narrow central reservation, high traffic density, and 30mph speed decreases from 62 to 52 and the associated willingness to pay decreases from $£ 1.59$ to $£ 1.32$ when we take into account the presence of a pedestrian refuge with 2 minutes waiting time and located 8 minutes away. 


\subsection{Number and value of new trips}

The barrier effect also affects the propensity to make trips that require walking across the road. This propensity can be derived from the estimated SP1 and SP3 models, as explained below.

The probability of choosing each of the three options in the SP1 exercise (A: Cross informally; B: Walk further to a location where the road goes through a tunnel; and C: Not making the trip) is the ratio between the exponentials of the utility of that option and the utilities of all three options (McFadden 1974). For example, the probabilities of Option A and $\mathrm{B}$ are given by the expressions below, where $U_{A l}, U_{B l}$, and $U_{C l}$ are the utilities of the three options, obtained by multiplying the relevant SP1 model coefficients by the specified values of the explanatory variables in expression 8.

$$
\begin{aligned}
& p_{A l}=\operatorname{EXP}\left(U_{A I}\right) /\left(\operatorname{EXP}\left(U_{A I}\right)+\operatorname{EXP}\left(U_{B I}\right)+\operatorname{EXP}\left(U_{C l}\right)\right) \\
& p_{B I}=\operatorname{EXP}\left(U_{B I}\right) /\left(\operatorname{EXP}\left(U_{A I}\right)+\operatorname{EXP}\left(U_{B I}\right)+\operatorname{EXP}\left(U_{C l}\right)\right)
\end{aligned}
$$

The probability of making the trip, in the choice scenario represented in SP1 is $\mathrm{P}_{\mathrm{A} 1+} \mathrm{P}_{\mathrm{B} 1}$. However, in the real world, the "walk further" option is not necessarily to walk to a place where the road goes through a tunnel but to walk to use a crossing facility. The probability of this option, for a given type of crossing facility, can be approximated by multiplying $\mathrm{p}_{\mathrm{B} 1}$ by the probability $p_{F A C}$ of choosing that type of crossing facility rather than not making the trip. The probability of making the trip then becomes

$$
p=p_{A I}+p_{B I}{ }^{*} p_{F A C}
$$

$p_{F A C}$ can be calculated from the SP3 model as below, where $U_{F A C}$ and $U_{D 3}$ are the utilities of using the specified facility and not making the trip respectively.

$$
p_{F A C}=E X P\left(U_{F A C}\right) /\left(E X P\left(U_{F A C}\right)+E X P\left(U_{D 3}\right)\right)
$$

The change in the propensity to walk across the road associated with a reduction in the barrier effect is then the change in the probability specified in expression 28 associated with changes in road characteristics or in the type or location of crossing facilities. Using the "rule of a half" (Williams 1977), the per-trip monetary value of the new trips generated from the reduction in the barrier effect can be approximated as half of the monetary value of that reduction for existing trips.

The overall monetary value of reducing the barrier effect (WTP), expressed as a proportion of the number of existing trips, can then be estimated as below, where $W T P_{C O M B}$ is the 
willingness to pay for reducing the barrier effect for existing trips and $\Delta p$ is the change in the propensity to make the trip, i.e. the difference in the value of $p$ in expression 28 after and before the reduction of the barrier effect.

$$
W T P=W_{C P M B}(1+0.5 \Delta p)
$$

As an example, the removal of the barrier effect of a road with 2 traffic lanes, a narrow central reservation, high traffic density, $30 \mathrm{mph}$ speed, and a pedestrian refuge with a 2 -minute wait and located 8 minutes away has a value of $£ 1.32$. This policy would generate $2.34 \%$ additional trips. The overall benefit, per existing trip, estimated by expression 30 would then be $£ 1.32 *(1+0.5 * 2.34 \%)=£ 1.34$

\section{Conclusions}

Road infrastructure and motorised traffic create a barrier effect on the movement of pedestrians, with possible negative implications on individuals and communities in the surrounding area. However, there are currently no robust quantitative methods to assess this effect. This paper has developed a comprehensive approach for the assessment of the barrier effect of roads on pedestrians and its integration in transport appraisal. An index was created to measure the barrier effect at a given point along a road, considering the design and traffic characteristics of the road and the characteristics and distance of the nearest pedestrian crossing facility. The index was then related with monetary values and with the estimated number of suppressed trips (i.e. the number of new trips that would be made if the barrier effect of the road was reduced), expressed as a proportion of the potential number of trips.

The approach produced robust results that take into account a range of hypothetical scenarios for the road, included in three stated preference exercises, while grounding the estimates in real-world behaviour, as assessed in a revealed preference exercise. The approach can be integrated into the appraisal of policy interventions that lead to reductions in severance, as it supports the estimation of the benefits of a wide range of improvements made to the road design, traffic and crossing facilities, while allowing for the disaggregation of those benefits by trip purpose.

The application of this approach in everyday appraisal requires further information to estimate, for example, the barrier effect of a whole section of a road, given the location of various crossing facilities along that section (the methods in this paper refer to the barrier effect felt at a single point along the road). The estimation of the total benefit of a policy intervention also requires information on the absolute change in the number of walking trips, 
which could be derived by multiplying the change in propensity to walk across the road by the maximum potential number of walking trips, considering the characteristics of local residents and the catchment areas of nearby trip attractors.

The range of attributes considered in this paper could be extended, by considering aspects such as traffic composition (in particular the proportion of large vehicles) and attributes related to the design and maintenance of crossing facilities (in particular footbridges and underpasses). The approach could also be adapted to assess the barrier effect felt by people walking along the road or using the road as a public place, which would require considering aspects such as quality of the pavements, landscaping, lighting, cleanliness, and other aspects of the road environment. A wider range of contexts could also be tested, considering weather conditions, presence of other pedestrians, and trips made by adults accompanying children.

The paper focused on generating overall estimates of the barrier effect but also provided estimates for different trip purposes. Additional segmentations could be defined with regards to the demographic characteristics of participants such as sex, age group, qualification levels, economic activity, car ownership, household composition, or disability (variables collected in our study). While the study included a question on income, it did not produce data that could be used in the analysis due to the large number of missing answers, although the results segmented for qualification levels could be a useful proxy.

Using a larger sample would also allow for the estimation of models that could capture how the magnitude and the value of the barrier effect varies with minor differences in attribute levels, including those that were not found to be statistically significant in this paper, such as the difference between crossing roads with a wide vs. narrow central reservation and with $10 \mathrm{mph}$ vs. $20 \mathrm{mph}$ vs. $30 \mathrm{mph}$ traffic speed.

A final question is the transferability of the estimates beyond the case study area. As mentioned in Section 2, the sample and population in one of the case study areas (Hull) have qualification levels slightly below the England national average, so the application of the estimates in areas with higher qualifications in England and in other countries may require an application of a correction factor. The applicability of the trade-off scenarios studied in this paper in other contexts should also take into consideration the variety of legal, social, and cultural aspects that influence the decision to cross the road in locations with no crossing facilities, such as fines and reluctance to challenge social norms. 


\section{Acknowledgements}

This project has received funding from Highways England. Thanks go to Chris Heywood (Accent) and Paul Metcalfe (PJM Economics).

\section{References}

Allen, M T., Austin, G W., Swaleheen, M. (2015) Measuring highway impacts on house prices using spatial regression. Journal of Sustainable Real Estate 7 (1), 83-98.

Anciaes, P R. (2015) What do we mean by "community severance"? Street Mobility and Network Accessibility Series, Working Paper 4, University College London, London., http://discovery.ucl.ac.uk/1527807

Anciaes, P R., Jones, P. (2018) A new method to value community severance caused by roads., in P Curtis, P Green, P Jones, P Anciaes (Eds.) Funding Sustainable Mobility and Liveability: Are the Current Scheme Appraisal Procedures Appropriate? CREATE project (Congestion Reduction in Europe: Advancing Transport Efficiency) Deliverable D5.2., Appendix C, pp.143-160.

Anciaes, P R., Boniface, S., Dhanani, A., Mindell, J S., Groce, N. (2016a) Urban transport and community severance: linking research and policy to link people and places. Journal of Transport and Health 3 (3), 268-277.

Anciaes, P R., Jones, P., Mindell, J M. (2016b) Community severance: where is it found and at what cost? Transport Reviews 36 (3), 293-317.

Anciaes, P R., Jones, P., Metcalfe, P. (2018) A stated preference model to value reductions in community severance caused by roads. Transport Policy 64, 10-19.

Anciaes, P R., Stockton, J., Ortegon, A., Scholes, S. (2019) Perceptions of road traffic conditions along with their reported impacts on walking are associated with wellbeing. Travel Behaviour and Society 15, 88-101.

Appleyard, D., Lintell, M. (1972) The environmental quality of city streets: the residents' viewpoint. Journal of the American Institute of Planners 38, 84-101.

ATAP (Australian Transport Assessment and Planning Steering Committee) (2018) Australian Transport Assessment and Planning Guidelines Part O3: Urban Amenity and Liveability. ATAP, Canberra., https://atap.gov.au/other-guidance/urban-amenity-liveability/files/o3-urban-amenity-liveability.pdf

Baltes, M., Chu, X. (2002) Pedestrian level of service for midblock street crossings. Transportation Research Record 1818, 125-133.

BMVI (Federal Ministry of Transport and Digital Infrastructure - Germany) (2016) Methodology Manual for the Federal Transport Infrastructure Plan 2030., http://www.bmvi.de/SharedDocs/EN/Documents/G/methodology-manual-for-the-ftip2030.pdf?_blob=publicationFile

Bosselmann, P., Macdonald, E., Kronemeyer, T. (1999) Livable streets revisited. Journal of the American Planning Association 65 (2), 168-180. 
Bretherton, W. M., Edwards, V., Miao, J. (2000) The economic impact of speed humps on housing values. ITE Journal 80 (1), 50-54.

Clark, J M., Hutton, B J., Burnett, N., Hathway, A., Harrison, A. (1991) The Appraisal of Community Severance. Transport and Road Research Laboratory, Crowthorne.

Ecoplan (2010) Manuel eNISTRA. Office Fédéral des Routes, Berne/Altdorf., http://www.astra.admin.ch/dienstleistungen/00129/00183/00187/index.html?lang=fr [in French]

Eliasson, J., Dillén, J L., Widell J. (2002) Measuring intrusion valuations through stated preferences and hedonic prices - a comparative study. AET Papers Repository., https://aetransport.org/public/downloads/ jdQO4/510-514ec4e74b792.pdf

Foley, L., Prins, R., Crawford, F., Sahlqvist, S., Ogilvie, D. (2017) Effects of living near a new urban motorway on the travel behaviour of local residents in deprived areas: Evidence from a natural experimental study. Health and Place 43, 57-65.

Garrod, G D., Scarpa, R., Willis, K G. (2002) Estimating the benefits of traffic calming on through routes: a choice experiment approach. Journal of Transport Economics and Policy 36 (2), 211-231.

Graham, J E., Jones, A T. (2019) A bump in the road: speed bumps' impact on property values. International Journal of Housing Markets and Analysis 12 (1), 43-58.

Granié, M-A., Brenac, T., Montel, M-C., Millot, M., Coquelet, C. (2014) Influence of built environment on pedestrian's crossing decision. Accident Analysis and Prevention 67, 75-85.

Grisolía, J M., López, F., Ortúzar, J D. (2015) Burying the highway: the social valuation of community severance and amenity. International Journal of Sustainable Transportation 9 (4), 298-309.

Handy, S. (2003) Amenity and severance., in D A Hensher and K J Button (Eds.) Handbook of Transport and the Environment. Elsevier, Amsterdam, pp. 117-140.

Héran, F. (2011) La Ville Morcelée. Effets de Coupure en Milieu Urbain [The fragmented city: barrier effects in urban contexts]. Economica, Paris [in French].

Hine, J. (1996) Pedestrian travel experiences - Assessing the impact of traffic on behaviour and perceptions of safety using an in-depth interview technique. Journal of Transport Geography 4, 179-199.

Jacobsen, P L., Racioppi, F., Rutter, H. (2009) Who owns the roads? How motorised traffic discourages walking and bicycling. Injury Prevention 15 (6), 369-373.

James, E., Millington, A., Tomlinson, P. (2005). Understanding community severance, Part 1: Views of practitioners and communities. Report for UK Department for Transport., http://webarchive.nationalarchives.gov.uk/+/http://www.dft.gov.uk/adobepdf/163944/Understanding_Co mmunity_Sev1.pdf

Kawamura, K., Mahajan, S. (2005) Hedonic analysis of impacts of traffic volumes on property values. Transportation Research Record 1924, 69-75.

Lassière, A. (1976) The Environmental Evaluation of Transport Plans. UK Department of Environment, London.

Lerväg, H. (1984) Vegen Som Barriere for Fotgjengere: Metodebeskrivelser [Roads as barriers for pedestrians: A description of methods]. NIBR Report 1984:13. NIBR, Oslo., http://urn.nb.no/URN:NBN:no-nb digibok 2012062608144 [in Norwegian]. 
Li, W., Saphores, J-D. (2012) Assessing impacts of freeway truck traffic on residential property values southern California case study. Transportation Research Record 2288, 48-56.

McFadden, D. (1974) Conditional logit analysis of qualitative choice behavior., in P Zarembka (Ed.) Frontiers in Econometrics. Academic Press, New York., pp.105-42.

McFadden, D., Train, K. (2000) Mixed MNL models of discrete response. Journal of Applied Econometrics 15 (5), 447-470.

Mindell, J S., Karlsen, S. (2012) Community severance and health: what do we actually know? Journal of Urban Health 89 (2), 232-246.

Mindell, J S., Anciaes, P R., Dhanani, A., Stockton, J., Jones, P., Haklay, M., Groce, M., Scholes, S., Vaughan., L. (2017) Using triangulation to assess a suite of tools to measure community severance. Journal of Transport Geography 60, 119-129.

MIT (Ministero delle Infrastrutture e dei Trasporti) [Ministry of Infrastructure and Transport - Italy]. (2008) Linee Guida Per La Misura Dei Costi Esterni Nell'ambito Del PON Trasporti 2000-2006 [Guidelines for the measurement of external costs as part of the 2000-2006 national operational programme for transport]. Quaderni del PON Trasporti 8., http://www.scuolavas.it/index.php/lgintroduzione/componenti-e-fattori-ambientali/mobilita-e-trasporti/viewdownload/10-mobilita-etrasporti/91-linee-guida-per-la-misura-dei-costi-esterni-nell-ambito-del-pon-trasporti-2000-2006-2008 [in Italian].

Nimegeer, A., Thomson, H., Foley, L., Hilton, S., Crawford, F., Ogilvie, D. (2018) Experiences of connectivity and severance in the wake of a new motorway: Implications for health and well-being. Social Science and Medicine 197, 78-86.

Rankavat, S., Tiwari, G. (2016) Pedestrians perceptions for utilization of pedestrian facilities - Delhi, India. Transportation Research Part F: Traffic Psychology and Behaviour 42 (3), 495-499.

Räsänen, M., Lajunen, T., Alticafarbay, F., Aydin, C. (2007) Pedestrian self-reports of factors influencing the use of pedestrian bridges. Accident Analysis and Prevention 39 (5), 969-973.

Read, M D., Cramphorn B. (2001) Quantifying the impact of social severance caused by roads. Research Report 201. Transfund New Zealand, Wellington.

Revelt, D., Train K E. (2000) Customer-specific taste parameters and mixed logit: households' choice of electricity supplier. Working Paper E00-275, Department of Economics, University of California at Berkeley., http://elsa.berkeley.edu/wp/train0999.pdf

Rose, J M., Bliemer, M C J. (2009) Constructing efficient stated choice experimental designs. Transport Reviews 29 (5), 587-617.

Russell, J., Hine, J. (1996) The impact of traffic on pedestrian behaviour - 1. Measuring the traffic barrier. Traffic Engineering and Control 37 (1), 16-18.

Sauter, D., Huettenmoser, M. (2008) Livable streets and social inclusion. Urban Design International 13 (2), 67-79.

Sillano, M., Ortúzar, J D. (2005) Willingness-to-pay estimation with mixed logit models: some new evidence. Environment and Planning A: Economy and Space 37 (3), 525-550. 
Soguel, N. C. (1995) Costing the traffic barrier: A contingent valuation survey. Environmental and Resource Economics 6, 301-308.

Tao, W., Mehndiratta, S., Deakin, E. (2010) Compulsory convenience? How large arterials and land use affect midblock crossing in Fushun, China. Journal of Transport and Land Use 3 (3), 61-82.

Tate, F N. (1995) The perception of danger from road traffic, in IPENZ (Ed.) Proceedings of the Institution of Professional Engineers New Zealand 1993: Sustainable Development. IPENZ, Wellington., pp.408419.

Tate, F N. (1997) Social severance. Research Report No.80. Transfund New Zealand, Wellington.

UK DfT (UK Department for Transport) (2017) Social Impact Appraisal, WebTAG Unit A4.1, https://www.gov.uk/government/publications/webtag-tag-unit-a4-1-social-impact-appraisal-december$\underline{2017}$

Van Eldijk, J. (2018) The wrong side of the tracks: quantifying barrier effects of transport infrastructure on local accessibility. Presented at European Transport Conference 2018., https://aetransport.org/en-gb/pastetc-papers/conference-papers-2018? abstractId $=6084 \&$ state $=$ b

Villaveces, A., Nieto, L.A., Ortega, D., Ríos, J.F., Medina, J.J., Gutiérrez, M.I., Rodríguez, D. (2012) Pedestrians' perceptions of walkability and safety in relation to the built environment in Cali, Colombia, 2009-10. Injury Prevention 18, 291-297.

Williams, H. (1977) On the formation of travel demand models and economic evaluation measure of user benefit. Environment and Planning 9, 285-344. 


\section{APPENDIX 1}

Indices of barrier effect and willingness to pay

ROAD TYPES

\begin{tabular}{|c|c|c|c|c|c|c|c|c|}
\hline \multirow[b]{2}{*}{$\begin{array}{l}\text { Central } \\
\text { reserv. }\end{array}$} & \multirow{2}{*}{$\begin{array}{l}\text { Traffic } \\
\text { density }\end{array}$} & \multirow{2}{*}{$\begin{array}{c}\text { Traffic } \\
\text { speed } \\
\text { (mph) }\end{array}$} & \multicolumn{2}{|c|}{ Roads with 1 lane } & \multicolumn{2}{|c|}{ Roads with 2 lanes } & \multicolumn{2}{|c|}{ Roads with 3 lanes } \\
\hline & & & Index & $\begin{array}{l}\text { Willingness } \\
\text { to pay (£) }\end{array}$ & Index & $\begin{array}{l}\text { Willingness } \\
\text { to pay (£) }\end{array}$ & Index & $\begin{array}{l}\text { Willingness } \\
\text { to pay }(\mathfrak{f})\end{array}$ \\
\hline \multirow{11}{*}{ Wide } & \multirow{4}{*}{ Low } & 10 & 0 & $£ 0.00$ & 21 & $£ 0.49$ & 48 & $£ 1.25$ \\
\hline & & 20 & 0 & $£ 0.00$ & 21 & $£ 0.49$ & 48 & $£ 1.25$ \\
\hline & & 30 & 3 & $£ 0.00$ & 23 & $£ 0.54$ & 50 & $£ 1.31$ \\
\hline & & 40 & 17 & $£ 0.37$ & 38 & $£ 0.96$ & 65 & $£ 1.72$ \\
\hline & \multirow{4}{*}{ medium } & 10 & 22 & $£ 0.52$ & 43 & $£ 1.11$ & 70 & $£ 1.87$ \\
\hline & & 20 & 22 & $£ 0.52$ & 43 & $£ 1.11$ & 70 & $£ 1.87$ \\
\hline & & 30 & 24 & $£ 0.58$ & 45 & $£ 1.16$ & 72 & $£ 1.93$ \\
\hline & & 40 & 39 & $£ 0.99$ & 60 & $£ 1.58$ & 87 & $£ 2.34$ \\
\hline & \multirow{3}{*}{ high } & 10 & 32 & $£ 0.82$ & 53 & $£ 1.40$ & 80 & $£ 2.17$ \\
\hline & & 20 & 32 & $£ 0.82$ & 53 & $£ 1.40$ & 80 & $£ 2.17$ \\
\hline & & 30 & 35 & $£ 0.87$ & 55 & $£ 1.46$ & 82 & $£ 2.22$ \\
\hline \multirow{11}{*}{ Narrow } & \multirow{4}{*}{ low } & 10 & 6 & $£ 0.00$ & 27 & $£ 0.61$ & 54 & $£ 1.38$ \\
\hline & & 20 & 6 & $£ 0.00$ & 27 & $£ 0.61$ & 54 & $£ 1.38$ \\
\hline & & 30 & 9 & $£ 0.00$ & 30 & $£ 0.67$ & 56 & $£ 1.43$ \\
\hline & & 40 & 24 & $£ 0.50$ & 44 & $£ 1.09$ & 71 & $£ 1.85$ \\
\hline & \multirow{4}{*}{ medium } & 10 & 28 & $£ 0.64$ & 49 & $£ 1.23$ & 76 & $£ 1.99$ \\
\hline & & 20 & 28 & $£ 0.64$ & 49 & $£ 1.23$ & 76 & $£ 1.99$ \\
\hline & & 30 & 31 & $£ 0.70$ & 51 & $£ 1.29$ & 78 & $£ 2.05$ \\
\hline & & 40 & 45 & $£ 1.12$ & 66 & $£ 1.71$ & 93 & $£ 2.47$ \\
\hline & \multirow{3}{*}{ high } & 10 & 38 & $£ 0.94$ & 59 & $£ 1.53$ & 86 & $£ 2.29$ \\
\hline & & 20 & 38 & $£ 0.94$ & 59 & $£ 1.53$ & 86 & $£ 2.29$ \\
\hline & & 30 & 41 & $£ 1.00$ & 62 & $£ 1.59$ & 89 & $£ 2.35$ \\
\hline \multirow{11}{*}{ No } & \multirow{4}{*}{ low } & 10 & 13 & $£ 0.23$ & 34 & $£ 0.81$ & 61 & $£ 1.58$ \\
\hline & & 20 & 13 & $£ 0.23$ & 34 & $£ 0.81$ & 61 & $£ 1.58$ \\
\hline & & 30 & 15 & $£ 0.28$ & 36 & $£ 0.87$ & 63 & $£ 1.63$ \\
\hline & & 40 & 30 & $£ 0.70$ & 51 & $£ 1.29$ & 78 & $£ 2.05$ \\
\hline & \multirow{4}{*}{ medium } & 10 & 35 & $£ 0.84$ & 56 & $£ 1.43$ & 83 & $£ 2.20$ \\
\hline & & 20 & 35 & $£ 0.84$ & 56 & $£ 1.43$ & 83 & $£ 2.20$ \\
\hline & & 30 & 37 & $£ 0.90$ & 58 & $£ 1.49$ & 85 & $£ 2.25$ \\
\hline & & 40 & 52 & $£ 1.32$ & 73 & $£ 1.91$ & 100 & $£ 2.67$ \\
\hline & \multirow{3}{*}{ high } & 10 & 45 & $£ 1.14$ & 66 & $£ 1.73$ & 93 & $£ 2.49$ \\
\hline & & 20 & 45 & $£ 1.14$ & 66 & $£ 1.73$ & 93 & $£ 2.49$ \\
\hline & & 30 & 47 & $£ 1.20$ & 68 & $£ 1.79$ & 95 & $£ 2.55$ \\
\hline
\end{tabular}

\section{CROSSING FACILITIES}

\begin{tabular}{lcccccccccccc}
\hline & \multicolumn{2}{c}{ No wait } & \multicolumn{2}{c}{ 30 seconds } & \multicolumn{2}{c}{ 60 seconds } & \multicolumn{1}{c}{ 120 seconds } & \multicolumn{2}{c}{ 180 seconds } & 240 seconds \\
\hline & Index & WTP & Index & WTP & Index & WTP & Index & WTP & Index & WTP & Index & WTP \\
\hline Pedestrian refuge & 8 & $£ 0.11$ & 9 & $£ 0.15$ & 10 & $£ 0.19$ & 12 & $£ 0.27$ & 14 & $£ 0.35$ & 16 & $£ 0.43$ \\
Straight signalised & 0 & $£ 0.00$ & 0 & $£ 0.00$ & 0 & $£ 0.00$ & 1 & $£ 0.00$ & 3 & $£ 0.03$ & 6 & $£ 0.11$ \\
Staggered signalised & 0 & $£ 0.00$ & 0 & $£ 0.00$ & 0 & $£ 0.00$ & 0 & $£ 0.00$ & 2 & $£ 0.00$ & 5 & $£ 0.07$ \\
Footbridge & 5 & $£ 0.05$ & & & & & & & & & & \\
High-quality footbridge & 0 & $£ 0.00$ & & & & & & & & & & \\
Underpass & 13 & $£ 0.29$ & & & & & & & & & & \\
\hline
\end{tabular}




\section{APPENDIX 2}

Indices of barrier effect and willingness to pay: by trip purpose

\section{WORK TRIPS}

\section{ROAD TYPES}

\begin{tabular}{|c|c|c|c|c|c|c|c|c|}
\hline \multirow[b]{2}{*}{$\begin{array}{l}\text { Central } \\
\text { reserv. }\end{array}$} & \multirow{2}{*}{$\begin{array}{l}\text { Traffic } \\
\text { density }\end{array}$} & \multirow{2}{*}{$\begin{array}{l}\text { Traffic } \\
\text { speed } \\
(\mathbf{m p h})\end{array}$} & \multicolumn{2}{|c|}{ Roads with 1 lane } & \multicolumn{2}{|c|}{ Roads with 2 lanes } & \multicolumn{2}{|c|}{ Roads with 3 lanes } \\
\hline & & & Index & $\begin{array}{l}\text { Willingness } \\
\text { to pay (£) }\end{array}$ & Index & $\begin{array}{l}\text { Willingness } \\
\text { to pay }(\mathfrak{£})\end{array}$ & Index & $\begin{array}{l}\text { Willingness } \\
\text { to pay (£) }\end{array}$ \\
\hline \multirow{11}{*}{ Wide } & \multirow{4}{*}{ Low } & 10 & 0 & $£ 0.00$ & 21 & $£ 0.45$ & 49 & $£ 1.13$ \\
\hline & & 20 & 0 & $£ 0.00$ & 21 & $£ 0.45$ & 49 & $£ 1.13$ \\
\hline & & 30 & 3 & $£ 0.00$ & 24 & $£ 0.52$ & 52 & $£ 1.20$ \\
\hline & & 40 & 17 & $£ 0.32$ & 38 & $£ 0.86$ & 66 & $£ 1.54$ \\
\hline & \multirow{4}{*}{ medium } & 10 & 22 & $£ 0.47$ & 43 & $£ 1.01$ & 71 & $£ 1.69$ \\
\hline & & 20 & 22 & $£ 0.47$ & 43 & $£ 1.01$ & 71 & $£ 1.69$ \\
\hline & & 30 & 25 & $£ 0.54$ & 46 & $£ 1.08$ & 74 & $£ 1.76$ \\
\hline & & 40 & 39 & $£ 0.88$ & 60 & $£ 1.42$ & 88 & $£ 2.10$ \\
\hline & \multirow{3}{*}{ high } & 10 & 32 & $£ 0.74$ & 53 & $£ 1.28$ & 81 & $£ 1.96$ \\
\hline & & 20 & 32 & $£ 0.74$ & 53 & $£ 1.28$ & 81 & $£ 1.96$ \\
\hline & & 30 & 35 & $£ 0.81$ & 56 & $£ 1.35$ & 84 & $£ 2.03$ \\
\hline \multirow{11}{*}{ Narrow } & \multirow{4}{*}{ low } & 10 & 6 & $£ 0.00$ & 27 & $£ 0.55$ & 55 & $£ 1.23$ \\
\hline & & 20 & 6 & $£ 0.00$ & 27 & $£ 0.55$ & 55 & $£ 1.23$ \\
\hline & & 30 & 9 & $£ 0.00$ & 30 & $£ 0.62$ & 58 & $£ 1.30$ \\
\hline & & 40 & 23 & $£ 0.42$ & 44 & $£ 0.96$ & 72 & $£ 1.64$ \\
\hline & \multirow{4}{*}{ medium } & 10 & 29 & $£ 0.57$ & 50 & $£ 1.11$ & 78 & $£ 1.79$ \\
\hline & & 20 & 29 & $£ 0.57$ & 50 & $£ 1.11$ & 78 & $£ 1.79$ \\
\hline & & 30 & 32 & $£ 0.64$ & 53 & $£ 1.18$ & 81 & $£ 1.86$ \\
\hline & & 40 & 45 & $£ 0.98$ & 66 & $£ 1.52$ & 94 & $£ 2.20$ \\
\hline & \multirow{3}{*}{ high } & 10 & 39 & $£ 0.84$ & 60 & $£ 1.38$ & 88 & $£ 2.05$ \\
\hline & & 20 & 39 & $£ 0.84$ & 60 & $£ 1.38$ & 88 & $£ 2.05$ \\
\hline & & 30 & 42 & $£ 0.91$ & 63 & $£ 1.45$ & 91 & $£ 2.12$ \\
\hline \multirow{11}{*}{ No } & \multirow{4}{*}{ low } & 10 & 15 & $£ 0.26$ & 36 & $£ 0.80$ & 64 & $£ 1.48$ \\
\hline & & 20 & 15 & $£ 0.26$ & 36 & $£ 0.80$ & 64 & $£ 1.48$ \\
\hline & & 30 & 18 & $£ 0.33$ & 39 & $£ 0.87$ & 67 & $£ 1.55$ \\
\hline & & 40 & 32 & $£ 0.67$ & 53 & $£ 1.21$ & 81 & $£ 1.89$ \\
\hline & \multirow{4}{*}{ medium } & 10 & 37 & $£ 0.82$ & 58 & $£ 1.36$ & 86 & $£ 2.04$ \\
\hline & & 20 & 37 & $£ 0.82$ & 58 & $£ 1.36$ & 86 & $£ 2.04$ \\
\hline & & 30 & 41 & $£ 0.89$ & 61 & $£ 1.43$ & 90 & $£ 2.11$ \\
\hline & & 40 & 54 & $£ 1.23$ & 75 & $£ 1.77$ & 100 & $£ 2.45$ \\
\hline & \multirow{3}{*}{ high } & 10 & 47 & $£ 1.09$ & 68 & $£ 1.63$ & 96 & $£ 2.31$ \\
\hline & & 20 & 47 & $£ 1.09$ & 68 & $£ 1.63$ & 96 & $£ 2.31$ \\
\hline & & 30 & 50 & $£ 1.16$ & 71 & $£ 1.70$ & 99 & $£ 2.38$ \\
\hline
\end{tabular}

\section{CROSSING FACILITIES}

\begin{tabular}{lcccccccccccc}
\hline & \multicolumn{2}{c}{ No wait } & \multicolumn{2}{c}{ 30 seconds } & \multicolumn{2}{c}{ 60 seconds } & \multicolumn{2}{c}{ 120 seconds } & \multicolumn{1}{c}{ 180 seconds } & 240 seconds \\
\hline & Index & WTP & Index & WTP & Index & WTP & Index & WTP & Index & WTP & Index & WTP \\
\hline Pedestrian refuge & 6 & $£ 0.12$ & 7 & $£ 0.17$ & 9 & $£ 0.22$ & 11 & $£ 0.31$ & 13 & $£ 0.41$ & 15 & $£ 0.51$ \\
Straight signalised & 0 & $£ 0.00$ & 0 & $£ 0.00$ & 0 & $£ 0.00$ & 2 & $£ 0.00$ & 4 & $£ 0.09$ & 6 & $£ 0.19$ \\
Staggered signalised & 0 & $£ 0.00$ & 0 & $£ 0.00$ & 0 & $£ 0.00$ & 1 & $£ 0.00$ & 3 & $£ 0.00$ & 6 & $£ 0.07$ \\
Footbridge & 2 & $£ 0.16$ & & & & & & & & & & \\
High-quality footbridge & 0 & $£ 0.05$ & & & & & & & & & & \\
Underpass & 11 & $£ 0.34$ & & & & & & & & & & \\
\hline
\end{tabular}




\section{SHOPPING TRIPS}

ROAD TYPES

\begin{tabular}{|c|c|c|c|c|c|c|c|c|}
\hline \multirow[b]{2}{*}{$\begin{array}{l}\text { Central } \\
\text { reserv. }\end{array}$} & \multirow[b]{2}{*}{$\begin{array}{l}\text { Traffic } \\
\text { density }\end{array}$} & \multirow{2}{*}{$\begin{array}{l}\text { Traffic } \\
\text { speed } \\
\text { (mph) }\end{array}$} & \multicolumn{2}{|c|}{ Roads with 1 lane } & \multicolumn{2}{|c|}{ Roads with 2 lanes } & \multicolumn{2}{|c|}{ Roads with 3 lanes } \\
\hline & & & Index & $\begin{array}{l}\text { Willingness } \\
\text { to pay (£) }\end{array}$ & Index & $\begin{array}{l}\text { Willingness } \\
\text { to pay }(\mathfrak{£})\end{array}$ & Index & $\begin{array}{l}\text { Willingness } \\
\text { to pay (f) }\end{array}$ \\
\hline \multirow{11}{*}{ Wide } & \multirow{4}{*}{ Low } & 10 & 0 & $£ 0.00$ & 21 & $£ 0.44$ & 46 & $£ 1.20$ \\
\hline & & 20 & 0 & $£ 0.00$ & 21 & $£ 0.44$ & 46 & $£ 1.20$ \\
\hline & & 30 & 2 & $£ 0.00$ & 23 & $£ 0.49$ & 48 & $£ 1.26$ \\
\hline & & 40 & 17 & $£ 0.32$ & 38 & $£ 0.93$ & 63 & $£ 1.69$ \\
\hline & \multirow{4}{*}{ medium } & 10 & 22 & $£ 0.48$ & 42 & $£ 1.09$ & 68 & $£ 1.85$ \\
\hline & & 20 & 22 & $£ 0.48$ & 42 & $£ 1.09$ & 68 & $£ 1.85$ \\
\hline & & 30 & 24 & $£ 0.53$ & 44 & $£ 1.14$ & 70 & $£ 1.90$ \\
\hline & & 40 & 39 & $£ 0.97$ & 59 & $£ 1.58$ & 85 & $£ 2.34$ \\
\hline & \multirow{3}{*}{ high } & 10 & 31 & $£ 0.77$ & 52 & $£ 1.38$ & 77 & $£ 2.14$ \\
\hline & & 20 & 31 & $£ 0.77$ & 52 & $£ 1.38$ & 77 & $£ 2.14$ \\
\hline & & 30 & 33 & $£ 0.82$ & 54 & $£ 1.43$ & 79 & $£ 2.19$ \\
\hline \multirow{11}{*}{ Narrow } & \multirow{4}{*}{ low } & 10 & 5 & $£ 0.00$ & 26 & $£ 0.60$ & 51 & $£ 1.36$ \\
\hline & & 20 & 5 & $£ 0.00$ & 26 & $£ 0.60$ & 51 & $£ 1.36$ \\
\hline & & 30 & 7 & $£ 0.00$ & 28 & $£ 0.65$ & 53 & $£ 1.41$ \\
\hline & & 40 & 22 & $£ 0.48$ & 43 & $£ 1.09$ & 68 & $£ 1.85$ \\
\hline & \multirow{4}{*}{ medium } & 10 & 27 & $£ 0.64$ & 47 & $£ 1.24$ & 73 & $£ 2.01$ \\
\hline & & 20 & 27 & $£ 0.64$ & 47 & $£ 1.24$ & 73 & $£ 2.01$ \\
\hline & & 30 & 29 & $£ 0.69$ & 49 & $£ 1.30$ & 75 & $£ 2.06$ \\
\hline & & 40 & 44 & $£ 1.13$ & 64 & $£ 1.73$ & 90 & $£ 2.50$ \\
\hline & \multirow{3}{*}{ high } & 10 & 36 & $£ 0.93$ & 57 & $£ 1.54$ & 82 & $£ 2.30$ \\
\hline & & 20 & 36 & $£ 0.93$ & 57 & $£ 1.54$ & 82 & $£ 2.30$ \\
\hline & & 30 & 38 & $£ 0.98$ & 59 & $£ 1.59$ & 84 & $£ 2.35$ \\
\hline \multirow{11}{*}{ No } & \multirow{4}{*}{ low } & 10 & 11 & $£ 0.13$ & 31 & $£ 0.73$ & 57 & $£ 1.50$ \\
\hline & & 20 & 11 & $£ 0.13$ & 31 & $£ 0.73$ & 57 & $£ 1.50$ \\
\hline & & 30 & 13 & $£ 0.18$ & 34 & $£ 0.79$ & 59 & $£ 1.55$ \\
\hline & & 40 & 28 & $£ 0.62$ & 49 & $£ 1.22$ & 74 & $£ 1.99$ \\
\hline & \multirow{4}{*}{ medium } & 10 & 32 & $£ 0.77$ & 53 & $£ 1.38$ & 79 & $£ 2.14$ \\
\hline & & 20 & 32 & $£ 0.77$ & 53 & $£ 1.38$ & 79 & $£ 2.14$ \\
\hline & & 30 & 35 & $£ 0.82$ & 55 & $£ 1.43$ & 81 & $£ 2.19$ \\
\hline & & 40 & 50 & $£ 1.26$ & 70 & $£ 1.87$ & 96 & $£ 2.63$ \\
\hline & \multirow{3}{*}{ high } & 10 & 42 & $£ 1.06$ & 62 & $£ 1.67$ & 88 & $£ 2.43$ \\
\hline & & 20 & 42 & $£ 1.06$ & 62 & $£ 1.67$ & 88 & $£ 2.43$ \\
\hline & & 30 & 44 & $£ 1.12$ & 65 & $£ 1.72$ & 90 & $£ 2.49$ \\
\hline
\end{tabular}

CROSSING FACILITIES

\begin{tabular}{lcccccccccccc}
\hline & \multicolumn{2}{c}{ No wait } & \multicolumn{2}{c}{ 30 seconds } & \multicolumn{2}{c}{ 60 seconds } & \multicolumn{1}{c}{ 120 seconds } & \multicolumn{1}{c}{ 180 seconds } & 240 seconds \\
\hline & Index & WTP & Index & WTP & Index & WTP & Index & WTP & Index & WTP & Index & WTP \\
\hline Pedestrian refuge & 7 & $£ 0.04$ & 9 & $£ 0.07$ & 10 & $£ 0.11$ & 13 & $£ 0.18$ & 15 & $£ 0.25$ & 18 & $£ 0.32$ \\
Straight signalised & 0 & $£ 0.00$ & 0 & $£ 0.00$ & 0 & $£ 0.00$ & 2 & $£ 0.00$ & 5 & $£ 0.00$ & 8 & $£ 0.02$ \\
Staggered signalised & 0 & $£ 0.00$ & 0 & $£ 0.00$ & 0 & $£ 0.00$ & 1 & $£ 0.00$ & 3 & $£ 0.00$ & 6 & $£ 0.00$ \\
Footbridge & 6 & $£ 0.00$ & & & & & & & & & & \\
High-quality footbridge & 6 & $£ 0.00$ & & & & & & & & & \\
Underpass & 16 & $£ 0.20$ & & & & & & & & & & \\
\hline
\end{tabular}




\section{LEISURE TRIPS}

ROAD TYPES

\begin{tabular}{|c|c|c|c|c|c|c|c|c|}
\hline \multirow{2}{*}{$\begin{array}{c}\text { Central } \\
\text { reserv. }\end{array}$} & \multirow{2}{*}{$\begin{array}{l}\text { Traffic } \\
\text { density }\end{array}$} & \multirow{2}{*}{$\begin{array}{l}\text { Traffic } \\
\text { speed } \\
\text { (mph) }\end{array}$} & \multicolumn{2}{|c|}{ Roads with 1 lane } & \multicolumn{2}{|c|}{ Roads with 2 lanes } & \multicolumn{2}{|c|}{ Roads with 3 lanes } \\
\hline & & & Index & $\begin{array}{l}\text { Willingness } \\
\text { to pay }(\mathfrak{f})\end{array}$ & Index & $\begin{array}{l}\text { Willingness } \\
\text { to pay }(\mathfrak{f})\end{array}$ & Index & $\begin{array}{l}\text { Willingness } \\
\text { to pay }(\mathfrak{f})\end{array}$ \\
\hline \multirow{11}{*}{ Wide } & \multirow{4}{*}{ Low } & 10 & 0 & $£ 0.00$ & 21 & $£ 0.57$ & 49 & $£ 1.37$ \\
\hline & & 20 & 0 & $£ 0.00$ & 21 & $£ 0.57$ & 49 & $£ 1.37$ \\
\hline & & 30 & 3 & $£ 0.00$ & 24 & $£ 0.62$ & 52 & $£ 1.43$ \\
\hline & & 40 & 18 & $£ 0.47$ & 39 & $£ 1.06$ & 67 & $£ 1.86$ \\
\hline & \multirow{4}{*}{ medium } & 10 & 22 & $£ 0.59$ & 43 & $£ 1.18$ & 71 & $£ 1.99$ \\
\hline & & 20 & 22 & $£ 0.59$ & 43 & $£ 1.18$ & 71 & $£ 1.99$ \\
\hline & & 30 & 25 & $£ 0.65$ & 46 & $£ 1.24$ & 74 & $£ 2.04$ \\
\hline & & 40 & 40 & $£ 1.08$ & 61 & $£ 1.67$ & 89 & $£ 2.48$ \\
\hline & \multirow{3}{*}{ high } & 10 & 33 & $£ 0.92$ & 54 & $£ 1.51$ & 82 & $£ 2.31$ \\
\hline & & 20 & 33 & $£ 0.92$ & 54 & $£ 1.51$ & 82 & $£ 2.31$ \\
\hline & & 30 & 36 & $£ 0.97$ & 57 & $£ 1.56$ & 85 & $£ 2.37$ \\
\hline \multirow{11}{*}{ Narrow } & \multirow{4}{*}{ low } & 10 & 7 & $£ 0.00$ & 28 & $£ 0.68$ & 56 & $£ 1.48$ \\
\hline & & 20 & 7 & $£ 0.00$ & 28 & $£ 0.68$ & 56 & $£ 1.48$ \\
\hline & & 30 & 10 & $£ 0.00$ & 31 & $£ 0.73$ & 59 & $£ 1.54$ \\
\hline & & 40 & 25 & $£ 0.57$ & 46 & $£ 1.16$ & 74 & $£ 1.97$ \\
\hline & \multirow{4}{*}{ medium } & 10 & 29 & $£ 0.70$ & 50 & $£ 1.29$ & 78 & $£ 2.10$ \\
\hline & & 20 & 29 & $£ 0.70$ & 50 & $£ 1.29$ & 78 & $£ 2.10$ \\
\hline & & 30 & 32 & $£ 0.76$ & 53 & $£ 1.35$ & 81 & $£ 2.15$ \\
\hline & & 40 & 47 & $£ 1.19$ & 69 & $£ 1.78$ & 96 & $£ 2.58$ \\
\hline & \multirow{3}{*}{ high } & 10 & 40 & $£ 1.02$ & 61 & $£ 1.61$ & 89 & $£ 2.42$ \\
\hline & & 20 & 40 & $£ 1.02$ & 61 & $£ 1.61$ & 89 & $£ 2.42$ \\
\hline & & 30 & 43 & $£ 1.08$ & 64 & $£ 1.67$ & 92 & $£ 2.48$ \\
\hline \multirow{11}{*}{ No } & \multirow{4}{*}{ low } & 10 & 14 & $£ 0.33$ & 35 & $£ 0.92$ & 63 & $£ 1.72$ \\
\hline & & 20 & 14 & $£ 0.33$ & 35 & $£ 0.92$ & 63 & $£ 1.72$ \\
\hline & & 30 & 16 & $£ 0.38$ & 38 & $£ 0.97$ & 65 & $£ 1.78$ \\
\hline & & 40 & 32 & $£ 0.81$ & 53 & $£ 1.40$ & 81 & $£ 2.21$ \\
\hline & \multirow{4}{*}{ medium } & 10 & 36 & $£ 0.94$ & 57 & $£ 1.53$ & 85 & $£ 2.34$ \\
\hline & & 20 & 36 & $£ 0.94$ & 57 & $£ 1.53$ & 85 & $£ 2.34$ \\
\hline & & 30 & 39 & $£ 1.00$ & 60 & $£ 1.59$ & 87 & $£ 2.39$ \\
\hline & & 40 & 54 & $£ 1.43$ & 75 & $£ 2.02$ & 100 & $£ 2.82$ \\
\hline & \multirow{3}{*}{ high } & 10 & 47 & $£ 1.27$ & 68 & $£ 1.85$ & 96 & $£ 2.66$ \\
\hline & & 20 & 47 & $£ 1.27$ & 68 & $£ 1.85$ & 96 & $£ 2.66$ \\
\hline & & 30 & 49 & $£ 1.32$ & 70 & $£ 1.91$ & 98 & $£ 2.72$ \\
\hline
\end{tabular}

CROSSING FACILITIES

\begin{tabular}{lcccccccccccc}
\hline & Index & WTP & Index & WTP & Index & WTP & Index & WTP & Index & WTP & Index & WTP \\
\hline Pedestrian refuge & 8 & $£ 0.20$ & 9 & $£ 0.24$ & 10 & $£ 0.28$ & 11 & $£ 0.36$ & 13 & $£ 0.44$ & 14 & $£ 0.51$ \\
Straight signalised & 0 & $£ 0.00$ & 0 & $£ 0.00$ & 0 & $£ 0.00$ & 0 & $£ 0.00$ & 1 & $£ 0.11$ & 3 & $£ 0.19$ \\
Staggered signalised & 0 & $£ 0.00$ & 0 & $£ 0.00$ & 0 & $£ 0.00$ & 0 & $£ 0.00$ & 1 & $£ 0.00$ & 2 & $£ 0.06$ \\
Footbridge & 4 & $£ 0.14$ & & & & & & & & & & \\
High-quality footbridge & 4 & $£ 0.15$ & & & & & & & & & & \\
Underpass & 13 & $£ 0.37$ & & & & & & & & & & \\
\hline
\end{tabular}

